\title{
OUTCOMES ASSOCIATED WITH OUTWARD BOUND AND NOLS PROGRAMS: A MEANS-END STUDY
}

\author{
A Thesis \\ presented to \\ the faculty of California Polytechnic State University, \\ San Luis Obispo
}

In Partial Fulfillment

Of the Requirements for the Degree

Master of Science in Agriculture, with Specialization in Recreation, Parks, and Tourism Management

By

Daniel Thomas Pronsolino

December 2009 
(C) 2009

Daniel Thomas Pronsolino

ALL RIGHTS RESERVED 


\section{COMMITTEE MEMBERSHIP}

TITLE:

Outcomes Associated with Outward Bound and NOLS Programs: A Means-end Study
AUTHOR:
Daniel Thomas Pronsolino

DATE SUBMITTED: $\quad$ December 2009

COMMITTEE CHAIR: Dr. Marni Goldenberg, Associate Professor, Recreation, Parks, and Tourism Administration Department

COMMITTEE MEMBER: Dr. Bill Hendricks, Department Head, Recreation, Parks, and Tourism Administration Department

COMMITTEE MEMBER: Daren Connor, Assistant Director of Programs, Associated Students, Inc. 


\section{DEDICATION}

This thesis is dedicated to my friends and family members who have encouraged me to continue pursuing my education during times of career uncertainty. Thank you to everyone who has supported me throughout this three-year process. 


\begin{abstract}
Outcomes Associated with Outward Bound and NOLS Programs: A Means-End Study Daniel T. Pronsolino
\end{abstract}

Outward Bound and the National Outdoor Leadership School (NOLS) are two of the United State's largest providers of outdoor education and adventure recreation programs. While many studies have examined the outcomes of the different organizations individually, there is very little comparative research. This study compared the attributes, consequences, and values obtained by 510 participants of courses 14 days or longer in the Rocky Mountain region during the summer of 2006.

Means-end theory was used to analyze data obtained from participants through personal interviews. Means-end theory links the physical objects or services, the means, with the outcomes and the personal values of the individual, the ends (Klenosky, Frauman, Norman, \& Gengler 1998). The theory focuses on the interrelationship among attributes, consequences, and values, as three levels of abstraction (Goldenberg, Klenosky, O’Leary, \& Templin, 2000).

Data were collected using a convenience sampling method from OB schools in Leadville, Marble, and Silverton, Colorado and the NOLS headquarters in Lander, Wyoming, for a total of 162 OB participants and 348 NOLS participants.

Interview questions were entered into LadderMap software, a program used to analyze means-end data. Content codes were developed and then tested by an additional researcher to measure intercoder reliability. An implication matrix was then created to tabulate the frequency of concepts being associated with one another. Hierarchical value 
maps (HVMs) were then created to graphically depict the themes and relationships that surfaced in the implication matrix.

Seven HVMs were developed to visually present the data for all participants, all NOLS participants, all OB participants, OB males, OB females, NOLS males, and NOLS females.

Though all HVMs were varied, some themes emerged by organization. For example, all NOLS participants had slightly more significant links to hard skills development than did their OB counterparts. NOLS and OB females stated being challenged and interactions as their most significant consequences yet corresponding males had slightly less emphasis on being challenged and more emphasis on new experience.

This study produced more similarities than differences among the various subsets of the population. For example all HVMs showed a clear link from multiple attributes to independence, and ultimately to transference and additional values. The HVMs showed that new experiences, being challenged, and group interactions were significant components for all participants. The most common values obtained also demonstrate great similarity among participant demographics. These values include transference, sense of accomplishment, self respect/esteem/confidence, and self-awareness.

Keywords: Means-end, Laddering, LadderMap, Outward Bound, National Outdoor Leadership School, NOLS, Outdoor Education, Outdoor Adventure, Wilderness Experience 


\section{ACKNOWLEDGEMENTS}

I would like to acknowledge the following individuals for their contributions to this project.

Dr. Marni Goldenberg, a very supportive and patient advisor. Dr Goldenberg introduced me to means-end theory and created the opportunity to be involved with the graduate program. Her continued guidance has led to the development of my own personal identity within the field of research. Her support and friendship have also been critical elements to the completion of this project.

Dr. Bill Hendricks and Darren Connor, for their continued support and patience as members of my thesis committee. Thank you for being so accommodating throughout this process.

Dr David Klenosky, of Purdue University, who went far above and beyond the call of duty by helping me navigate the trials and tribulations of 15 year old software programs for data analysis. Dr. Klenosky is a truly passionate researcher whose generosity and expertise has made this project possible.

The staff of Outward Bound and NOLS who not only allowed the study to occur but also provided great support for this project throughout the years and hospitality during the summer of data collection.

The USDA McIntire-Stennis and ARI Grant programs who provided the funding for this initial collection of data, as well as the future longitudinal study. Without the funding of organizations like these, it would not be possible to advance academic research. 


\section{TABLE OF CONTENTS}

PAGE

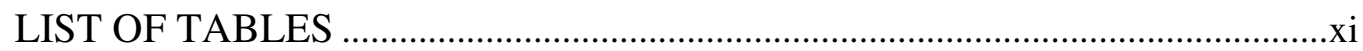

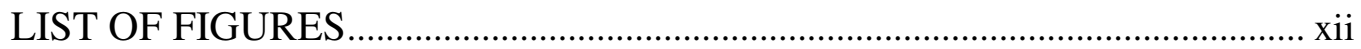

CHAPTER 1 INTRODUCTION ..............................................................

Background of the Study .................................................................

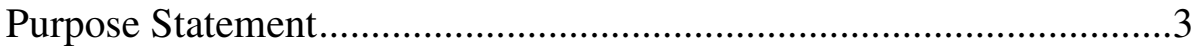

Research Questions ................................................................... 3

Professional Significance .......................................................... 3

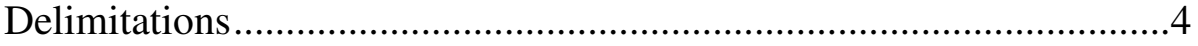

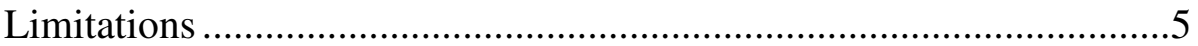

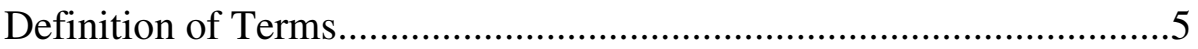

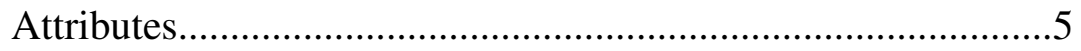

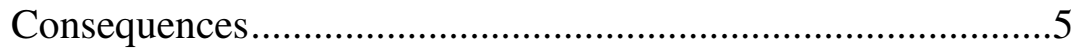

Hierarchical Value Map (HVM) .........................................

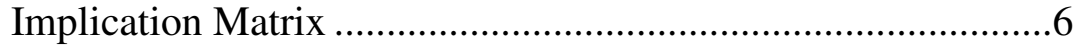

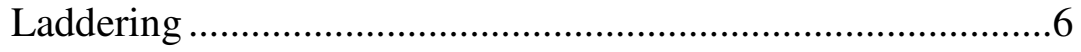

LadderMap .................................................................6

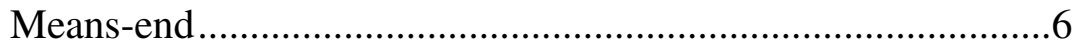

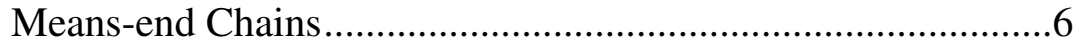

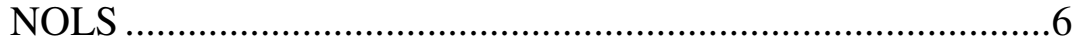

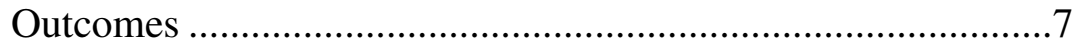

Outward Bound ................................................................

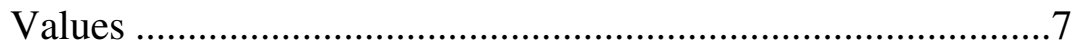

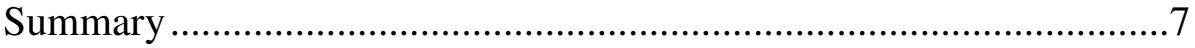

CHAPTER 2 REVIEW OF LITERATURE .................................................

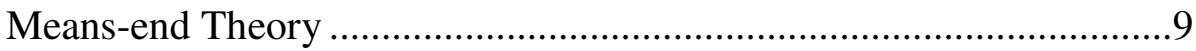

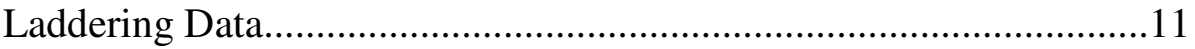

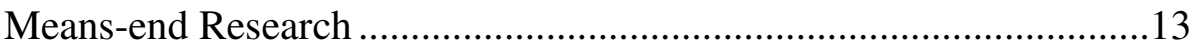

Longitudinal Studies ..............................................................22

Outdoor Education ................................................................23

Outdoor Adventure Recreation Programs.......................................25

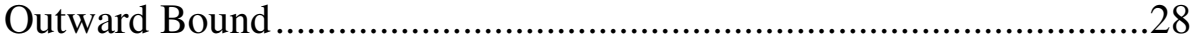

National Outdoor Leadership Schools (NOLS) ................................30

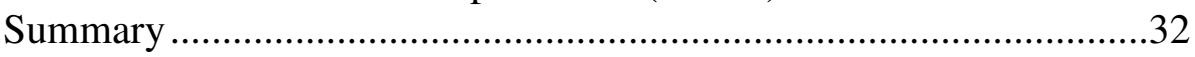

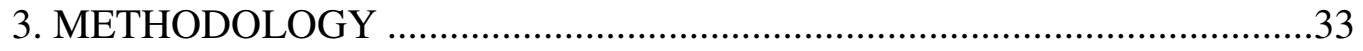




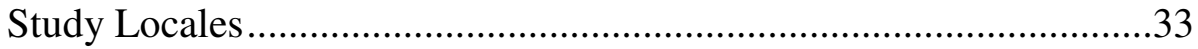

Research Participants ...................................................................34

Instruments Used in Data Collection .................................................34

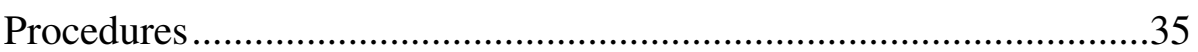

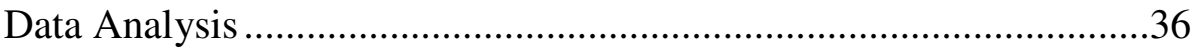

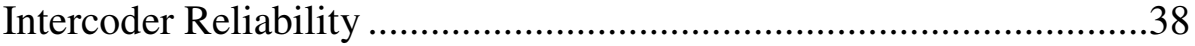

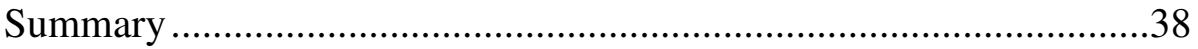

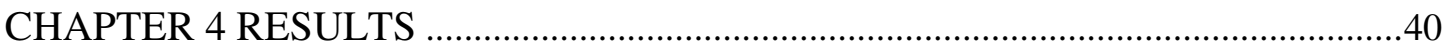

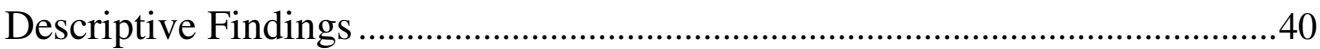

Respondents by Organization ..................................................................40

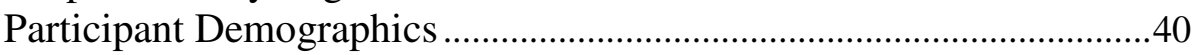

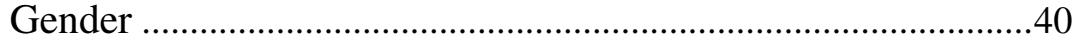

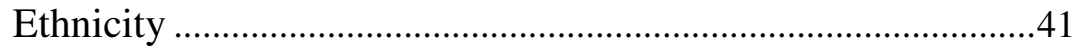

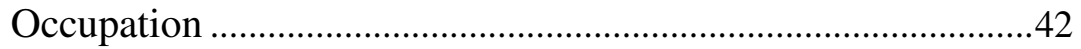

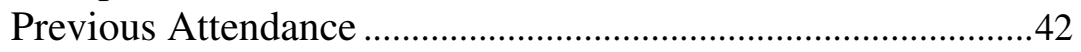

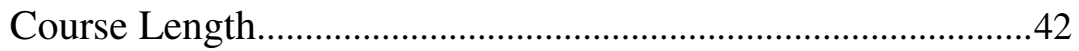

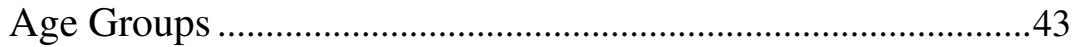

Reccomending OB/NOLS to a friend........................................43

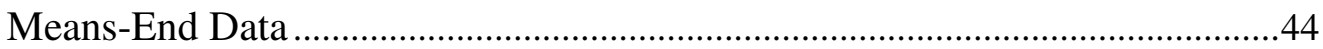

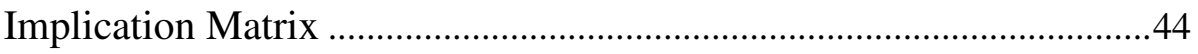

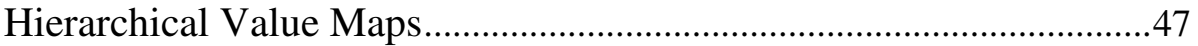

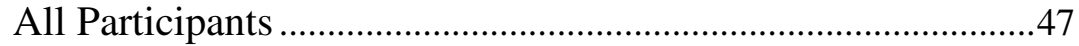

All NOLS Participants..................................................................50

All OB Participants ....................................................................52

NOLS Females ............................................................................54

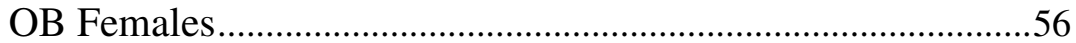

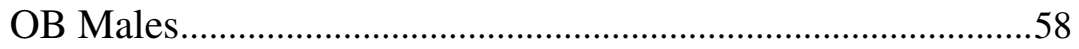

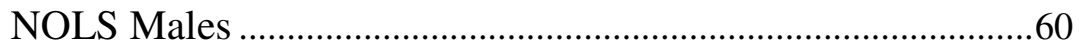

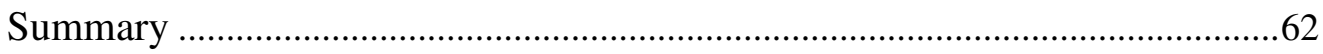

CHAPTER 5 SUMMARY, DISCUSSION, IMPLICATIONS, AND

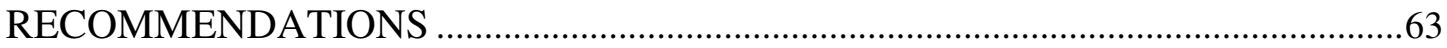

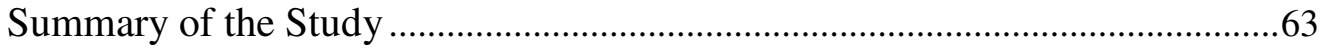

Summary of Data Procedures ................................................................64

Summary of Significant Findings ..........................................................65

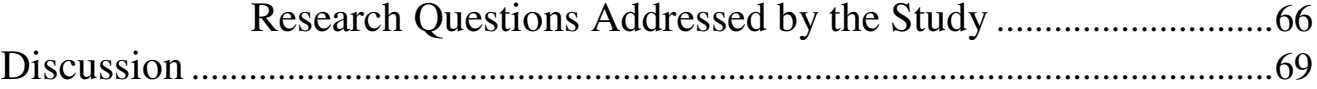

Outdoor Education and Adventure Recreation Literature ........69

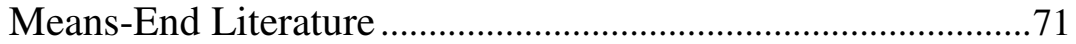

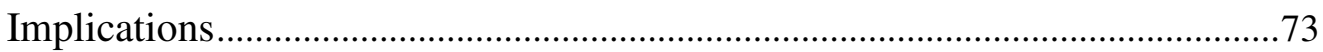




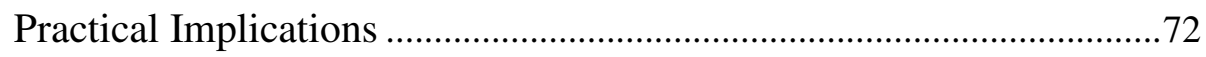

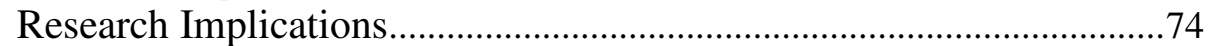

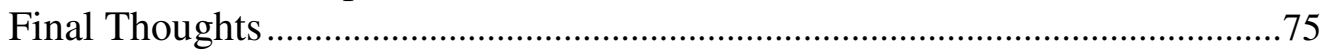

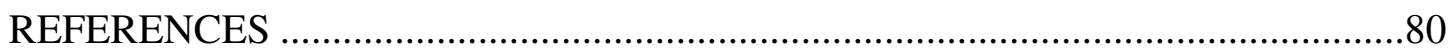

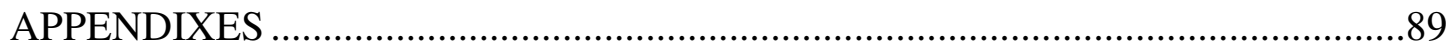

Appendix A: Interview Script ......................................................90

Appendix B: Cover Letter.........................................................95

Appendix C: Parental Consent Form ...............................................96

Appendix D: Alphabetical List of Content Codes and Definitions .....97

Appendix E: Content Codes by Frequency....................................100 


\section{LIST OF TABLES}

Table 2.1. Means-End Investigations Prior to 2003 .........................................16

Table 2.2. Means-End Investigations, 2003-2006 .........................................20

Table 2.3. Outward Bound Core Values .........................................................28

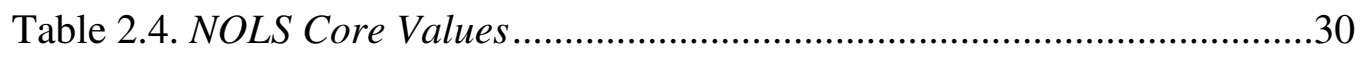

Table 4.1. Respondents by Organization ....................................................41

Table 4.2. Respondents by Ethnic/Racial Group ............................................41

Table 4.3. Course Length ............................................................................42

Table 4.4. Respondents by Age Group ..........................................................43

Table 4.5. Implication Matrix ...............................................................46

Table 4.6. Cutoff Levels and Percent Associations for HVMs.........................47 


\section{LIST OF FIGURES}

Figure 4.1. Hierarchical Value Map for all Participants .......................................49

Figure 4.2. Hierarchical Value Map for all NOLS Participants ...............................51

Figure 4.3. Hierarchical Value Map for all $O B$ Participants..................................53

Figure 4.4. Hierarchical Value Map for NOLS Female Participants.........................55

Figure 4.5. Hierarchical Value Map for OB Female Participants .............................57

Figure 4.6. Hierarchical Value Map for OB Male Participants ................................59

Figure 4.7. Hierarchical Value Map for NOLS Male Participants ...........................61 


\section{Chapter 1}

\section{INTRODUCTION}

This thesis is a comparison of long term outcomes associated with participation in Outward Bound (OB) and National Outdoor Leadership Schools (NOLS) courses. This chapter presents the background of the study, the problem statement, professional significance of the study, an overview of the methodology, delimitations of the study, and concludes with a list of key terms.

\section{Background of the Study}

Means-end theory, developed by Gutman (1982), has been used in several studies of consumer purchasing behavior (Baker, Thompson, \& Engelken, 2002; Futopoulos, Krystallis, \& Ness, 2003; Klenosky, Gengler, \& Mulvey, 1993; Walker \& Olson, 1991) and many others. The theory has seen some limited application to outdoor programming such as understanding the outcomes associated with ropes course programming (Goldenberg et al., 2000; Haras, Bunting, \& Witt, 2006) and examining the components of an Outward Bound experience (Goldenberg, McAvoy, \& Klenosky, 2005). Other means-end studies have examined ecotourists' preference for interpretive programs (Klenosky et al., 1998), and explored factors of greenway/trail use (Frauman \& Cunningham, 2001). These studies have demonstrated the potential of means-end theory in examining the outcomes associated with participation in outdoor experiences. This study seeks to expand the current base of means-end knowledge to include a comparison 
of outcomes associated with participation in two of the United States leading outdoor program providers, OB and NOLS.

For the purposes of this study, outcomes are defined as the end result in the participants' minds. This includes participants' perceptions of both positive and negative consequences of their involvement in an outdoor experience, and any values they feel they have obtained directly through the course which may impact their lives in the future.

Both OB and NOLS have a long tradition in the outdoor education field. OB was founded in 1941 in Great Britain by Kurt Hahn, one of the pioneers of both outdoor education and experiential education fields (Outward Bound USA, n.d.). OB currently operates outdoor programs for participants of all ages at a multitude of sites in the US and internationally varying in course length from one day to an entire semester (Outward Bound USA, n.d.).

NOLS was founded by the legendary outdoor educator Paul Petzoldt in 1965 in Lander, Wyoming. Since its original summer of 100 all-male participants, NOLS has graduated over 75,000 coed participants. NOLS currently operates 11 schools worldwide and is considered by many to be the world leader in outdoor education (National Outdoor Leadership School, n.d.).

NOLS has traditionally been thought of as more technical skills based than OB, largely due to Pezholdt's original goal of training future outdoor educators to lead trips for organizations such as $\mathrm{OB}$. As both organizations have evolved over the years, their curriculum has become more similar but there is very limited prior research to determine any similarities or differences in their outcomes. 


\section{Purpose Statement}

The purpose of this study was to compare the attributes, consequences, and values associated with participation in OB and NOLS courses using means-end theory. A secondary purpose was to collect data for future longitudinal analyses.

The study specifically examined the subjects' links between the components of the OB or NOLS courses they participated in (the means), and the benefits or values they received from the course (the ends). Means-end theory was used to analyze the linkages between these two concepts. This study also sought to analyze the differences between subgroups of the population separated gender, OB participants, and NOLS participants.

\section{Research Questions}

This study addresses the following research questions:

1. What are the attributes, consequences, and values associated with participation in OB and NOLS adventure recreation courses?

2. What are the means-end relationships between the attributes, consequences, and values?

3. What are the differences in means-end structures between participants of different genders and programs?

\section{Professional Significance}

This study seeks to expand the current body of means-end knowledge by applying the theory to a longitudinal study. Recent applications of means-end theory in outdoor education programs (Frauman \& Cunningham, 2001; Goldenberg et al., 2005; 
Goldenberg et al., 2000; Klenosky et al., 1998) have demonstrated the ability of meansend to serve as a useful tool in the outdoor field but none have applied the theory to a longitudinal study.

This study also adds to the body of knowledge on OB and NOLS programs as well as the greater industry of outdoor education. Many studies have documented outcomes associated with outdoor education programs such as leadership, self-concept, academic achievement, personality development, interpersonal skills, and becoming more adventurous (Hattie, Marsh, Neill, \& Richards, 1997), but there is limited comparative research on outcomes from OB and NOLS.

This study also provides practical information for both OB and NOLS to use in the training of staff and marketing of future courses.

\section{Delimitations}

The study was delimited to the following parameters:

1. The study was conducted during the summer of 2006.

2. The study analyzed self-reported outcomes of participation in NOLS and OB courses.

3. Subjects were participants of OB and NOLS courses ages 14 and over.

4. Courses were selected based on convenience, ease of access, and a minimum duration of 14 days.

5. Interviews were conducted at base camp locations on the final day of the course.

6. A convenience sample methodology was used to select courses and individual participants. 


\section{Limitations}

This study was limited by the following factors:

1. Semi-structured interviews were conducted in varying settings influenced by such factors as weather, meals, presence of additional group members, and general distraction from course-end activities.

2. Content codes were established in order to group participant responses into like categories.

3. A large sample size $(n=510)$ was utilized and provided challenges for LadderMap software which has primarily been used for sample sizes of less than 100 .

\section{Definition of Terms}

Attributes. The physical objects, services, or experiences of the individual, typically viewed as being relatively concrete (Goldenberg, 2002). Attributes for this study were specific components of outdoor recreation programs.

Consequences. The direct result of attributes whether positive or negative. Negative consequences are referred to as costs or risks, positive consequences are frequently referred to as benefits (Goldenberg, 2002).

Hierarchical Value Map (HVM). A visual illustration of the relationships between concepts by showing the links between the attributes, consequences, and values. In an HVM, each attribute, consequence, and value appears in a circle, color coded accordingly. The lines connecting the circles depict the frequency of that link by the 
thickness of the line. In other words, the more frequently two concepts are linked by subjects, the thicker the line between the concepts in the HVM.

Implication Matrix. "An asymmetric matrix that summarizes the number of times each category concept implies or leads to the other concepts in respondents' ladders" (Klenosky et al., 1998, p. 27).

Laddering. A method of means-end data collection that builds means-end chains by asking a participant why an attribute is important, the response is either another attribute or a consequence. The researcher then asks the participant why their response is important to them, and then why the next response is important to them, and then why the next response is important to them, until the participant eventually reaches a value. In this method, each response is similar to a rung on a ladder, eventually leading the researcher to the top level of the participants' thinking, the value.

LadderMap. A software program developed by Gengler and Reynolds (1995) that helps analyze means-end data and create implication matrices and hierarchical value maps.

Means-end. A theory used to analyze consumer purchasing behavior through examining links between attributes, consequences, and values associated with a product.

Means-end Chains. The linkages between the attributes, consequences, and values.

NOLS. National Outdoor Leadership School, which is a provider of outdoor adventure education programs with 11 campuses worldwide. 
Outcomes. The end results of the course, as perceived in the participants' minds. This includes any positive or negative consequences of their involvement and any values they feel they have gained.

Outward Bound $(\mathrm{OB})$. A provider of outdoor education and outdoor adventure education programs with over 40 campus worldwide.

Values. The desired end state in the participants' minds. These are the participants' end destination as they travel up the means-end ladder of abstraction from the more concrete attributes to the highly abstract value-states (Klenosky et al., 1993).

\section{Summary}

In summary, this research was designed to examine and compare the outcomes of NOLS and OB programs. Using means-end theory, laddering techniques and LadderMap software, the outcomes were analyzed and linked to course components. The secondary purpose of this research was to create a body of data that can be used for future longitudinal analyses.

This thesis was organized into 5 chapters. Chapter 2 is the review of literature which attempts to encompass all previous research relevant to theoretical framework and the appropriate fields of study. The literature review discusses means-end theory, its prior application and methods, outdoor education, outdoor adventure recreation programming, Outward Bound, and NOLS.

Chapter three gives an in-depth view of the methodology used for the study. This chapter outlines the purpose of the study, research context, research participants, instruments used, procedures, data analysis, and intercoder reliability. 
Chapter four provides detailed results of the study presented in both narrative and table formats. Descriptive statistics such as participant demographics are presented first followed by means-end data. The means-end data includes an analysis of the content codes developed, frequency of the content codes being mentioned, and an implication matrix displaying the links between content codes. The final means-end section presents the hierarchical value maps and narrative descriptions to further explain their findings. Chapter five presents the discussion, implications, and recommendations for future research. The chapter begins with summaries of the purpose of the study, procedures, and data analysis. The research questions for the study are then answered in detail followed by a discussion of how the findings compare with published literature. This section is broken into two subcategories: outdoor education and adventure recreation literature, and means-end literature. The chapter concludes with the practical and research implications followed by the researcher's final thoughts. 


\section{CHAPTER 2}

\section{REVIEW OF LITERATURE}

The purpose of this chapter is to examine the existing literature and theoretical frameworks relating to means-end theory, outdoor education, and outdoor adventure recreation programs. This review has been divided into the following categories: meansend theory, applying means-end theory, previous means-end studies, longitudinal studies, outdoor education, outdoor adventure recreation programs, Outward Bound, and NOLS.

\section{Means-End Theory}

Means-end theory was developed by Gutman (1982) as a method of analyzing the factors driving consumer purchasing behavior. The theory views consumers as goaloriented decision makers who are motivated to choose behaviors that will lead to specific desirable outcomes (Costa \& Dekker, 2004). This is not unlike Expectancy-value theory which states that consumer actions produce consequences and that consumers learn to associate specific consequences with particular aspects of a product (Gutman \& Miaoulis, 2003). Both theories examine the process of how consumers develop an opinion about a product or service and how that process leads to the intended outcome, but means-end theory goes beyond the direct consequences and continues to the more abstract values associated with those consequences. Means-end theory links the physical objects or tangible services being examined, the means, with the direct outcomes and any personal values that may be obtained, the ends (Klenosky et al., 1998). The theory focuses on the 
interrelationship among attributes, consequences, and values, as three levels of abstraction (Goldenberg et al., 2000).

The attributes are the physical objects, services, or experiences of the individual (Goldenberg, 2002). Attributes of an outdoor education experience could include group activities, instruction, or specific components such as rafting, kayaking, or backpacking.

Consequences are the direct result of attributes whether positive or negative. Negative consequences are referred to as costs or risks, positive consequences are frequently referred to as benefits. Some examples of positive consequences or benefits of an outdoor education experience could include participants developing technical skills, learning teamwork, or developing interpersonal skills. Some possible negative consequences could include injury, feeling homesick, or physical exhaustion.

The values are the desired end state in the participants' minds. These are the participants' end destination as they travel up the means-end ladder of abstraction from the more concrete attributes to the highly abstract value-states (Klenosky et al., 1993).

The linkages between the attributes, consequences, and values are described as means-end chains. Each link in the means-end chain describes how a participant's thoughts have progressed from either attribute to consequence or consequence to value so that the reader can clearly follow the thought process from start to finish. For example, a means-end chain for an outdoor education experience could include the attribute "first aid training." This attribute could then be linked to the consequence "teamwork," which could be linked to the value "compassion." In other words, this hypothetical participant has indicated that first aid training helped build teamwork which led to a feeling of compassion for the group. 


\section{Laddering Data}

Means-end chains are constructed by a data collection technique known as laddering. Laddering first conceived by Olson and Reynolds (1983) and further developed in theory and application by Reynolds and Gutman (1988). Laddering builds means-end chains by asking a participant why an attribute is important, the response is either another attribute or a consequence. The researcher then asks the participant why their response is important to them, and then why the next response is important to them, until the participant eventually reaches a value. In this method, each response is similar to a rung on a ladder, eventually leading the researcher to the top level of the participants' thinking, the value.

Collection of means-end data has been done by personal interview (Gutman, 1982; Olson \& Reynolds, 1983; Hofstede, Audenaert, Steenkamp, \& Wedel, 1998; Klenosky, Templin, \& Troutman 2001; Klenosky, 2002; Reynolds \& Gutman, 1988; Reynolds \& Rochon, 1991; Roth, 1994;), by telephone interview (Bagozzi \& Dobholkar, 1994), and by self-administered questionnaire (Frauman \& Cunningham, 2001; Goldenberg et al., 2000; Pieters, Botschen, \& Thelen, 1998; Walker \& Olson, 1991 ). Personal interviews have remained the most frequent method of collecting means-end data. The use of questionnaires has proven very effective when attempting to utilize a large sample size or when financial or geographical barriers prevent the researcher from being in direct contact with the subjects (Goldenberg, 2002; McAvoy, Holman, Goldenberg, \& Klenosky, 2006).

Once the data have been collected, whether by interview or questionnaire, it is entered into a software program called LadderMap (Gengler \& Reynolds, 1995). While 
being entered, each participant response is coded by the researcher to categorize responses. Once all data have been entered and coded, a second researcher conducts a blind review to test intercoder reliability.

Once the data have been tested for intercoder reliability, LadderMap software is used to compile an implication matrix to examine the frequency of links between concepts. "The coded elements of each ladder are aggregated across subjects and used to develop an implication matrix - an asymmetric matrix that summarizes the number of times each category concept implies or leads to the other concepts in respondents' ladders" (Klenosky et al., 1998, p. 27). Once the implication matrix has been completed, the data can then be used to construct hierarchical value maps (HVMs). HVMs visually illustrate the relationships between concepts by showing the links between the attributes, consequences, and values. HVMs can be constructed to represent the entire data set, or any data subsets of interest to the researcher. In an HVM, each attribute, consequence, and value appears in a circle, color coded accordingly. The lines connecting the circles depict the frequency of that link by the thickness of the line. In other words, the more frequently two concepts are linked by subjects, the thicker the line between the concepts in the HVM.

Early laddering data were an immense task to process and greatly increased the cost of studies using laddering as a collection method (Gengler \& Reynolds, 1995). In 1995, an MSDOS software package was developed by Gengler and Reynolds (1995) to help with data processing. This program, known as LadderMap, helps researchers by sorting data into content codes and providing a quantitative assessment of all paired relationships or links between concepts (Gengler \& Reynolds, 1995). One of the most 
useful applications of LadderMap is its ability to create Hierarchical Value Maps (HVMs) from the data. Automated creation of HVMs allows researchers to create individual HVMs for each subset of data in a very short period of time compared to manual construction and tabulation.

\section{Means-end Research}

Means-end theory was originally developed for consumer marketing by Gutman (1982). For several years, means-end was used primarily in a marketing and consumer choice context (Grunert \& Grunert, 1995). Some marketing examples include Olson and Reynolds' (1983) development of a means-end model for advertising using a nonalcoholic beverage example, Hotstede, Steenkamp, and Wedel's (1999) examination of cross-national market segments, Baker et al., (2002) comparison of organic food purchasing habits between Germany and Britain, and Skytte and Bove's (2004) study of wholesale purchasing of pork and fish products. Costa, Dekker, and Jongen (2004) examined previous means-end research in the context of consumer food product choice and discovered limitations in the application of means-end to consumer decision making behavior. Their review suggested statistical models for future use specifically in the field of food related product marketing. For a complete list of previous means-end studies prior to 2003, see Table 2.1, means-end studies from 2003 to 2006 can be found in Table 2.2 .

The first use of means-end theory outside of the advertising industry was a study of ethical behavior of sales personnel (Pitts, Wong, \& Whalen, 1991). This was the first demonstration of using means-end for non-consumer based decision making behavior and 
began a series of studies broadening the uses of means-end. Similar studies examined recycling behavior (Bagozzi \& Dobholkar, 1994), health care policy issues (Roth, 1994), motivation and behavior patterns of museum visitors (Jansen-Verbeke \& van Rekon, 1996), and public perceptions of President Clinton (Bagozzi \& Dabholkar, 2000).

Means-end theory first appeared in the field of recreation research with a study of ski destination choices (Klenosky et al., 1993). Other recreation based means-end studies have included consumers' involvement with tennis rackets (Mulvey, Olson, Celsi, \& Walker, (1994), tourist selection of interpretive programs (Klenosky et al., 1998), greenway/trail benefits and personal values (Frauman \& Cunningham, 2001), student athletes' college selection decisions (Klenosky et al., 2001), push and pull factors in travel decision making (Klenosky, 2002) and wilderness participation for those with and without disabilities (McAvoy et al., 2006).

Other studies that have dealt with outdoor education and adventure recreation organizations include ropes course participation (Goldenberg et al., 2000). This study compared attributes, consequences, and values of ropes course participation and found similarities among values obtained suggesting common themes of self worth and selffulfillment, derived from participation. Haras et al. (2006) expanded the use of meansend theory in ropes course participation by comparing two approaches to program delivery, challenge by choice, and inviting optimum participation. Results of the Haras et al. study found that the use of different approaches allowed facilitators to manipulate and maximize participant benefits.

Goldenberg et al. (2005) examine outcomes associated with specific components of an Outward Bound experience. The Goldenberg et al. study used self-administered 
questionnaires to analyze the outcomes of specific course components of OB programs such as climbing, or backpacking. The perceived outcomes reported by participants demonstrated the ability to link course experiences with personal values that could be transferred beyond the course. These values were perceived by participants as a positive contribution to their lives. The Goldenberg et al. study provided a reference point for OB programs but did not compare outcomes among other providers of similar outdoor education experiences.

A very similar study was conducted in 2006 using personal interviews for an evaluation of a service component of Outward Bound (Goldenberg, Pronsolino, \& Klenosky, 2006). Aside from the use of personal interviews, this study mirrored the approach of Goldenberg's 2005 OB study and specifically examined the course component of service. Both the 2005 and 2006 studies provided similar lists of attributes, consequences, and values that were examined the creation of content codes for this study.

When compared with this study, the smaller sample size of Goldenberg et al. (2006) study (n=78), was able to produce more coherent HVM's representing very high percentages of the associations between attributes, consequences, and values. The Goldenberg et al. study also examined service from the perspective of OB employees, making it a study of observations, rather than a study of participants' perceived personal outcomes. 
Table 2.1. Means-End Investigations Prior to 2003: Reproduced with permission from Goldenberg (2002).

\begin{tabular}{|c|c|c|c|}
\hline Author(s) \& date & $\begin{array}{l}\text { Study focus \& } \\
\text { product/setting involved }\end{array}$ & $\begin{array}{l}\text { Approach used } \\
\text { in study }\end{array}$ & Sample \\
\hline Gutman (1982) & $\begin{array}{l}\text { Introduced means-end } \\
\text { theory and the ideas } \\
\text { underlying the analysis of } \\
\text { means-end data. Used } \\
\text { example involving } \\
\text { breakfast beverage } \\
\text { products. }\end{array}$ & Interviews & Not reported \\
\hline $\begin{array}{l}\text { Olson \& Reynolds } \\
\text { (1983) }\end{array}$ & $\begin{array}{l}\text { Demonstrated laddering } \\
\text { technique and data analysis } \\
\text { procedures; and introduced } \\
\text { the "MECCAS Model" } \\
\text { (Means-End } \\
\text { Conceptualization of } \\
\text { Components for } \\
\text { Advertising Strategy) to } \\
\text { show how the means-end } \\
\text { approach could be used to } \\
\text { develop advertising } \\
\text { strategy. Presented example } \\
\text { involving non-alcoholic } \\
\text { beverage products. }\end{array}$ & Interviews & Not reported \\
\hline $\begin{array}{l}\text { Reynolds \& } \\
\text { Gutman (1988) }\end{array}$ & $\begin{array}{l}\text { Demonstrated theory, } \\
\text { methods, and analytical } \\
\text { techniques for conducting } \\
\text { laddering interviews. } \\
\text { Presented example } \\
\text { involving wine-cooler } \\
\text { products. }\end{array}$ & Interviews & Not reported \\
\hline $\begin{array}{l}\text { Pitts, Wong, \& } \\
\text { Whalen (1991) }\end{array}$ & $\begin{array}{l}\text { Means-end study of } \\
\text { ethical/unethical behavior } \\
\text { of sales personnel. }\end{array}$ & $\begin{array}{l}\text { Presented } \\
\text { scenarios, } \\
\text { responded to } \\
\text { questions }\end{array}$ & $\begin{array}{l}n=257 \\
\text { undergraduate } \\
\text { students }\end{array}$ \\
\hline
\end{tabular}




\begin{tabular}{|c|c|c|c|}
\hline $\begin{array}{l}\text { Reynolds \& } \\
\text { Rochon (1991) }\end{array}$ & $\begin{array}{l}\text { Demonstrated an } \\
\text { application of the } \\
\text { "MECCAS Model" } \\
\text { (Means-End } \\
\text { Conceptualization of } \\
\text { Components for } \\
\text { Advertising Strategy). } \\
\text { Means-end study of } \\
\text { message elements in beer } \\
\text { advertisements. }\end{array}$ & Interviews & $\begin{array}{l}n=42 \\
\text { consumers }\end{array}$ \\
\hline $\begin{array}{l}\text { Walker \& Olson } \\
\text { (1991) }\end{array}$ & $\begin{array}{l}\text { Explored how means-end } \\
\text { approach could be used to } \\
\text { understand consumers' } \\
\text { product involvement. } \\
\text { Means-end study of } \\
\text { greeting card selection. }\end{array}$ & Questionnaires & $\begin{array}{l}n=40 \text { female } \\
\text { college students }\end{array}$ \\
\hline $\begin{array}{l}\text { Klenosky, } \\
\text { Gengler, \& } \\
\text { Mulvey (1993) }\end{array}$ & $\begin{array}{l}\text { Demonstrated means-end } \\
\text { approach in a recreation } \\
\text { choice context. Means-end } \\
\text { study of ski destination } \\
\text { choice. }\end{array}$ & Interviews & $\begin{array}{l}n=90 \text { ski trade } \\
\text { show attendees }\end{array}$ \\
\hline $\begin{array}{l}\text { Spreng \& } \\
\text { Olshavsky (1993) }\end{array}$ & $\begin{array}{l}\text { Disconfirmation model with } \\
\text { consumer satisfaction } \\
\text { looking at new camera. }\end{array}$ & $\begin{array}{l}\text { Small group, } \\
\text { worked on } \\
\text { booklets }\end{array}$ & $\begin{array}{l}n=128 \text { college } \\
\text { students }\end{array}$ \\
\hline $\begin{array}{l}\text { Bagozzi \& } \\
\text { Dobholkar (1994) }\end{array}$ & $\begin{array}{l}\text { Used means-end approach } \\
\text { to examine recycling } \\
\text { behavior. }\end{array}$ & $\begin{array}{l}\text { Telephone } \\
\text { interviews }\end{array}$ & $\begin{array}{l}n=130, \text { random } \\
\text { sample of city } \\
\text { residents }\end{array}$ \\
\hline $\begin{array}{l}\text { Mulvey, Olson, } \\
\text { Celsi, \& Walker } \\
\text { (1994) }\end{array}$ & $\begin{array}{l}\text { Means-end study of } \\
\text { consumers' involvement } \\
\text { with tennis rackets. }\end{array}$ & Questionnaires & $\begin{array}{l}n=58 \\
\text { undergraduate } \\
\text { students } \\
\text { (beginner, } \\
\text { intermediate } \\
\text { and expert } \\
\text { tennis players) }\end{array}$ \\
\hline Roth (1994) & $\begin{array}{l}\text { A means-end study of three } \\
\text { health care policy issues. }\end{array}$ & Interviews & $\begin{array}{l}n=45 \text { health } \\
\text { care consumers }\end{array}$ \\
\hline $\begin{array}{l}\text { Pieters, } \\
\text { Baumgartner, \& } \\
\text { Allen (1995) }\end{array}$ & $\begin{array}{l}\text { Showed how means-end } \\
\text { theory could be used to } \\
\text { study consumers' goals - } \\
\text { specifically weight loss } \\
\text { goals. }\end{array}$ & Questionnaires & $\begin{array}{l}n=51 \\
\text { undergraduate } \\
\text { students }\end{array}$ \\
\hline
\end{tabular}




\begin{tabular}{|c|c|c|c|}
\hline $\begin{array}{l}\text { Jansen-Verbeke \& } \\
\text { van Rekom (1996) }\end{array}$ & $\begin{array}{l}\text { A means-end study of } \\
\text { motivation and behavior } \\
\text { patterns of museum visitors. }\end{array}$ & $\begin{array}{l}\text { Interviews } \\
\text { Questionnaires }\end{array}$ & $\begin{array}{l}n=30 \text { visitors to } \\
\text { an art museum }\end{array}$ \\
\hline $\begin{array}{l}\text { Barczak, Ellen, \& } \\
\text { Pilling (1997) }\end{array}$ & $\begin{array}{l}\text { Means-end study of } \\
\text { technology-based banking } \\
\text { services. }\end{array}$ & $\begin{array}{l}\text { Interviews } \\
\text { Focus groups } \\
\text { Questionnaire }\end{array}$ & $\begin{array}{l}n=13 \\
n=32 \\
n=331 \\
\text { customers from } \\
\text { a mid-size } \\
\text { metropolitan } \\
\text { bank }\end{array}$ \\
\hline Graeff (1997) & $\begin{array}{l}\text { Used means-end approach } \\
\text { to examine consumers' } \\
\text { product inferences and } \\
\text { attitudes. Means-end study } \\
\text { focusing on camera } \\
\text { advertisements. }\end{array}$ & Questionnaire & $\begin{array}{l}n=120 \\
\text { consumers }\end{array}$ \\
\hline $\begin{array}{l}\text { Botschen, \& } \\
\text { Hemetsberger } \\
(1998)\end{array}$ & $\begin{array}{l}\text { Means-end study of } \\
\text { consumer perceptions of a } \\
\text { specific clothing line across } \\
\text { three countries. }\end{array}$ & Questionnaires & $\begin{array}{l}n=1,081 \\
\text { consumers of a } \\
\text { manufacturer of } \\
\text { high branded } \\
\text { clothes }\end{array}$ \\
\hline $\begin{array}{l}\text { Hofstede, } \\
\text { Audenaert, } \\
\text { Steenkamp, \& } \\
\text { Wedel (1998) }\end{array}$ & $\begin{array}{l}\text { Explored an alternative } \\
\text { approach, the Association } \\
\text { Pattern Technique (APT), } \\
\text { for demonstrating means- } \\
\text { end relationships. Study } \\
\text { involved Belgium food } \\
\text { products. }\end{array}$ & Interviews & $\begin{array}{l}n=300 \\
\text { consumers }\end{array}$ \\
\hline $\begin{array}{l}\text { Klenosky, } \\
\text { Frauman, Norman, } \\
\text { \& Gengler (1998) }\end{array}$ & $\begin{array}{l}\text { Means-end study of tourist } \\
\text { selection of interpretive } \\
\text { programs. }\end{array}$ & Interviews & $\begin{array}{l}n=47 \text { state park } \\
\text { visitors }\end{array}$ \\
\hline $\begin{array}{l}\text { Pieters, Botschen, } \\
\& \text { Thelen (1998) }\end{array}$ & $\begin{array}{l}\text { Means-end study of } \\
\text { expectations of clothing } \\
\text { store service employees. }\end{array}$ & Questionnaires & $\begin{array}{l}n=231 \\
\text { undergraduate } \\
\text { students }\end{array}$ \\
\hline $\begin{array}{l}\text { Hotstede, } \\
\text { Audenaert, } \\
\text { Steenkamp, \& } \\
\text { Wedel (1999) }\end{array}$ & $\begin{array}{l}\text { Means-end study of cross- } \\
\text { national market segments. }\end{array}$ & $\begin{array}{l}\text { International } \\
\text { survey }\end{array}$ & $\begin{array}{l}n=2961 \\
\text { consumers from } \\
11 \text { countries }\end{array}$ \\
\hline $\begin{array}{l}\text { Langerak, Peelen, } \\
\& \text { Nijssen (1999) }\end{array}$ & $\begin{array}{l}\text { Means-end study of new } \\
\text { product development } \\
\text { lifecycles. }\end{array}$ & $\begin{array}{l}\text { Performed } \\
\text { tasks }\end{array}$ & $\begin{array}{l}n=20 \text { product } \\
\text { development } \\
\text { experts }\end{array}$ \\
\hline
\end{tabular}




\begin{tabular}{|c|c|c|c|}
\hline $\begin{array}{l}\text { Bagozzi \& } \\
\text { Dabholkar (2000) }\end{array}$ & $\begin{array}{l}\text { Means-end study of public } \\
\text { perceptions of President } \\
\text { Clinton. }\end{array}$ & Interviews & $\begin{array}{l}n=603, \text { random } \\
\text { sample }\end{array}$ \\
\hline $\begin{array}{l}\text { Goldenberg, } \\
\text { Klenosky, } \\
\text { O'Leary, \& } \\
\text { Templin (2000) }\end{array}$ & $\begin{array}{l}\text { Demonstrated how means- } \\
\text { end approach could be used } \\
\text { to study participation in a } \\
\text { recreation activity. Means- } \\
\text { end study of ropes course } \\
\text { participation. }\end{array}$ & Questionnaires & $\begin{array}{l}n=125 \text { ropes } \\
\text { course } \\
\text { participants, } 18- \\
50 \text { years old }\end{array}$ \\
\hline $\begin{array}{l}\text { Herrmann, Huber, } \\
\& \text { Braustein (2000) }\end{array}$ & $\begin{array}{l}\text { Means-end study of internal } \\
\text { quality and external } \\
\text { customer needs and } \\
\text { satisfaction. }\end{array}$ & Interviews & $\begin{array}{l}n=186 \\
\text { consumers }\end{array}$ \\
\hline Ligas (2000) & $\begin{array}{l}\text { Means-end study of } \\
\text { consumer-product } \\
\text { relationships/motives } \\
\text { behind purchasing a new } \\
\text { house within the last three } \\
\text { years. }\end{array}$ & Interviews & $\begin{array}{l}n=4 \text { single } \\
\text { women }\end{array}$ \\
\hline $\begin{array}{l}\text { Collen \& Hoekstra } \\
(2001)\end{array}$ & $\begin{array}{l}\text { Looks at feasibility of } \\
\text { research using means-end } \\
\text { theory to uncover values } \\
\text { used as determinants of } \\
\text { preferences for housing } \\
\text { attributes. }\end{array}$ & Interviews & $\begin{array}{l}n=10 \text { pilot } \\
\text { study, } 60 \text { to } \\
\text { follow in sequel } \\
\text { article }\end{array}$ \\
\hline $\begin{array}{l}\text { Frauman \& } \\
\text { Cunningham } \\
(2001)\end{array}$ & $\begin{array}{l}\text { Examined means-end } \\
\text { relationships among the } \\
\text { Greenway/trail benefits and } \\
\text { personal values. }\end{array}$ & Questionnaires & $\begin{array}{l}n=270 \\
\text { greenway trail } \\
\text { users }\end{array}$ \\
\hline $\begin{array}{l}\text { Garbarino \& } \\
\text { Johnson (2001) }\end{array}$ & $\begin{array}{l}\text { Looked at consumers' goal } \\
\text { orientation and its effects } \\
\text { on overall satisfaction and } \\
\text { product usage in theater } \\
\text { goers. }\end{array}$ & Surveys & $\begin{array}{l}n=378 \text { theater } \\
\text { goers }\end{array}$ \\
\hline $\begin{array}{l}\text { Jaeger \& MacFie } \\
(2001)\end{array}$ & $\begin{array}{l}\text { Examined the effects of } \\
\text { advertising content and } \\
\text { format on consumer } \\
\text { expectations of new apple } \\
\text { varieties. }\end{array}$ & Interviews & $\begin{array}{l}n=169 \text { regular } \\
\text { consumers of } \\
\text { apples }\end{array}$ \\
\hline
\end{tabular}




\begin{tabular}{|c|c|c|c|}
\hline $\begin{array}{l}\text { Klenosky, } \\
\text { Templin, \& } \\
\text { Troutman (2001) }\end{array}$ & $\begin{array}{l}\text { Means-end study of } \\
\text { student-athletes' college } \\
\text { selection decisions. }\end{array}$ & Interviews & $\begin{array}{l}n=27 \text { NCAA } \\
\text { Division I } \\
\text { collegiate } \\
\text { football players }\end{array}$ \\
\hline $\begin{array}{l}\text { Baker, Thompson, } \\
\text { \& Engelken (2002) }\end{array}$ & $\begin{array}{l}\text { Use means-end to map the } \\
\text { values of organic food } \\
\text { choice in the UK compared } \\
\text { to Germany. }\end{array}$ & Interviews & $\begin{array}{l}n=64 \text { regular } \\
\text { consumers of } \\
\text { organic foods, } \\
32 \text { from UK and } \\
32 \text { from } \\
\text { Germany }\end{array}$ \\
\hline Klenosky (2002) & $\begin{array}{l}\text { Demonstrated how means- } \\
\text { end approach can be used to } \\
\text { examine the relationship } \\
\text { among push and pull factors } \\
\text { in travel decision making. } \\
\text { Means-end study of spring } \\
\text { break destination choice. }\end{array}$ & Interviews & $\begin{array}{l}n=53 \text { college } \\
\text { students }\end{array}$ \\
\hline
\end{tabular}

Table 2.2. Means-End Literature from 2003-2006

\begin{tabular}{|c|c|c|c|}
\hline $\begin{array}{l}\text { Devlin, Birtwistle, } \\
\text { \& Macedo (2003) }\end{array}$ & $\begin{array}{l}\text { Use means-end chain analysis } \\
\text { to examine food retail } \\
\text { positioning strategy. }\end{array}$ & Interviews & $\begin{array}{l}n=15 \\
\text { individuals } \\
\text { responsible for } \\
\text { shopping for } \\
\text { their household }\end{array}$ \\
\hline $\begin{array}{l}\text { Futopoulos, } \\
\text { Krystallis, \& Ness } \\
(2003)\end{array}$ & $\begin{array}{l}\text { Means-end chain analysis } \\
\text { used to uncover organic } \\
\text { buyers purchasing motives } \\
\text { compared to non-buyers in } \\
\text { the organic grape industry in } \\
\text { Greece. }\end{array}$ & Interviews & $\begin{array}{l}n=28 \text { organic } \\
\text { food buyers } \\
\text { and } 21 \text { organic } \\
\text { food non- } \\
\text { buyers }\end{array}$ \\
\hline $\begin{array}{l}\text { Gutman \& Miaoulis } \\
\text { (2003) }\end{array}$ & $\begin{array}{l}\text { Using means-end theory to } \\
\text { create a marketing strategy } \\
\text { for a university. }\end{array}$ & Interviews & $\begin{array}{l}n=86 \text { first year } \\
\text { students }\end{array}$ \\
\hline $\begin{array}{l}\text { Orsingher \& } \\
\text { Marzocchi (2003) }\end{array}$ & $\begin{array}{l}\text { Uses the hierarchical } \\
\text { representation of satisfied } \\
\text { consumer service experience } \\
\text { to identify links and } \\
\text { consumer values. }\end{array}$ & Questionnaire & $\begin{array}{l}n=85 \text { satisfied } \\
\text { customers of a } \\
\text { large hotel }\end{array}$ \\
\hline Wansink (2003) & $\begin{array}{l}\text { Using means-end ladders to } \\
\text { understand brand equity and }\end{array}$ & Interviews & $n=1200$ \\
\hline
\end{tabular}




\begin{tabular}{|c|c|c|c|}
\hline & $\begin{array}{l}\text { its implications on a } \\
\text { marketing campaign. }\end{array}$ & & consumers \\
\hline $\begin{array}{l}\text { Costa, Dekker, \& } \\
\text { Jongen (2004) }\end{array}$ & $\begin{array}{l}\text { Examines the strengths and } \\
\text { shortcomings of means-end } \\
\text { theory in the context of } \\
\text { consumer-oriented food } \\
\text { product choice. }\end{array}$ & $\begin{array}{l}\text { Literature } \\
\text { review }\end{array}$ & \\
\hline $\begin{array}{l}\text { Mort \& Rose } \\
(2004)\end{array}$ & $\begin{array}{l}\text { Product type and its effects } \\
\text { on linkages produced in } \\
\text { means-end chains. }\end{array}$ & Questionnaire & $\begin{array}{l}n=191 \\
\text { customers in } \\
\text { large shopping } \\
\text { area in } \\
\text { Australia }\end{array}$ \\
\hline $\begin{array}{l}\text { Leppard, Russell, \& } \\
\text { Cox (2003) }\end{array}$ & $\begin{array}{l}\text { Demonstrates the "top down } \\
\text { cut-off" method of laddering } \\
\text { means-end data. }\end{array}$ & $\begin{array}{l}\text { Used data from } \\
\text { previous study } \\
\text { utilizing } \\
\text { interviews and } \\
\text { questionnaires }\end{array}$ & $\begin{array}{l}n=244 \\
\text { Australian and } \\
\text { Malaysian } \\
\text { Mothers }\end{array}$ \\
\hline $\begin{array}{l}\text { Skytte \& Bove } \\
(2004)\end{array}$ & $\begin{array}{l}\text { Examines retailers purchasing } \\
\text { of pork and fish products and } \\
\text { the related attributes, } \\
\text { consequences and values } \\
\text { when buying for their } \\
\text { company. }\end{array}$ & Interviews & $\begin{array}{l}n=46 \text { retailers } \\
\text { from Germany } \\
\& 25 \text { retailers } \\
\text { from Denmark }\end{array}$ \\
\hline $\begin{array}{l}\text { Russell, Busson, } \\
\text { Flight, Bryan, Van } \\
\text { Lawik Van Pabst, } \\
\text { \& Cox (2004) }\end{array}$ & $\begin{array}{l}\text { Compared effectiveness of } \\
\text { interviews verses } \\
\text { questionnaires. }\end{array}$ & $\begin{array}{l}\text { Used data from } \\
\text { previous study } \\
\text { utilizing } \\
\text { interviews and } \\
\text { questionnaires }\end{array}$ & $\begin{array}{l}n=244 \\
\text { Australian and } \\
\text { Malaysian } \\
\text { Mothers }\end{array}$ \\
\hline $\begin{array}{l}\text { Brunso, Scholderer, } \\
\text { \& Grunert (2004) }\end{array}$ & $\begin{array}{l}\text { Used means-end approach to } \\
\text { examine lifestyle decision } \\
\text { making. }\end{array}$ & Questionnaires & $\begin{array}{l}n=1000 \\
\text { French } \\
\text { Consumers }\end{array}$ \\
\hline $\begin{array}{l}\text { Grunert, \& Bech- } \\
\text { Larsen (2004) }\end{array}$ & $\begin{array}{l}\text { Means-end study of } \\
\text { consumers' purchasing } \\
\text { decisions of organic verses } \\
\text { traditional pork. }\end{array}$ & Interviews & $n=60$ \\
\hline $\begin{array}{l}\text { McIntosh, \& Thyne } \\
\text { (2005) }\end{array}$ & $\begin{array}{l}\text { A published review of } \\
\text { literature of means-end } \\
\text { research in tourism }\end{array}$ & N/A & N/A \\
\hline $\begin{array}{l}\text { Goldenberg, } \\
\text { McAvoy, \& } \\
\text { Klenosky (2005) }\end{array}$ & $\begin{array}{l}\text { Means-end analysis of } \\
\text { outcomes of Outward Bound } \\
\text { experiences }\end{array}$ & Questionnaires & $\begin{array}{l}n=216 \\
\text { Outward Bound } \\
\text { Participants }\end{array}$ \\
\hline Goldenberg, & Means-end evaluation of a & Personal & $N=78$ Outward \\
\hline
\end{tabular}




\begin{tabular}{|c|c|c|c|}
\hline $\begin{array}{l}\text { Pronsolino, \& } \\
\text { Klenosky (2006) }\end{array}$ & $\begin{array}{l}\text { service component in } \\
\text { Outward Bound programs }\end{array}$ & Interviews & $\begin{array}{l}\text { Bound } \\
\text { Employees }\end{array}$ \\
\hline
\end{tabular}

\section{Longitudinal Studies}

Longitudinal studies involve multiple data collections from the same participants on the same research topics over time. An important distinction between longitudinal and retroactive is that retroactive studies collect data about past events at a single point in time. Longitudinal studies collect data during an event and repeatedly after the event to track changes over time (Ware, 1985) This allows conclusions to be drawn about longterm impacts of the issue being studied by examining multiple data points for each participant and each research question. Longitudinal studies can be utilized in any field interested in studying impacts over time but they have extensive implications in medical and behavioral studies (Ware, 1985).

The main benefit of longitudinal studies is their ability to document actual results over time without the need to make speculative models for predicting future behavior (Diggle, Liang, \& Zeger, 1994). Utilizing a longitudinal study, service providers can follow-up with clients and understand the impact of their services throughout the duration of the study. This helps understand how the subjects' responses evolve over the months or years and allows the researcher to track changes. This is an important difference compared to a one time or point-in-time study which only capture subjects' responses at a single time and doesn't reveal changing trends.

One of the drawbacks of longitudinal studies is the extensive amount of resources involved in conducting them. Researchers have to plan and conduct multiple data 
collections over a period of time that could be 30 years or more for some studies. This requires extensive time, financial, and personnel resources. For longer studies, accuracy can also be compromised by replacement of research personnel. Another concern is maintaining contact information and involvement from participants. Management of contact information is critical to maintaining a large-enough sample size (Ware, 1985).

There are many factors that can influence the sample size that is maintained throughout a study. The initial collection of contact information is perhaps the most important. Collecting as many forms of contact information as possible can be helpful but losing contact with some participants is inevitable. Many factors in the subjects' lives can also influence loss of contact such as age, career status, marital status, military service, or death (Diggle et al., 1994).

Like any form of research, longitudinal studies have certain circumstances where they are appropriate and can offer valuable information in a variety of fields of study. Within the field of outdoor research, longitudinal studies have been conducted on the effects of ropes course participation on group cohesion (Meyer \& Wenger, 1998).

\section{Outdoor Education}

Outdoor education has had a variety of definitions which all share the central ideas of teaching and learning in and about the outdoors. Many researchers have established more formal definitions such as "the use of experiences in the outdoors for the education and development of the whole person" (The Outdoor Institute, 2004, II 6), or "a means of curriculum enrichment, whereby the process of learning takes place out of doors. Outdoor education broadly includes environmental education, 
conservation education, adventure education, school camping, wilderness therapy, and some aspects of outdoor recreation” (Lappin, 2000, I 2). One appealingly concise definition was presented by Ford. "Outdoor education is education 'in', 'about', and 'for' the out-of-doors" (Ford, 1981, p. 12).

The concept of outdoor education first emerged in the United States as "school camping." The term "school camping" didn't resonate with the public who considered recreational activities such as camping as an inappropriate use of taxpayer money. To preserve the programs and maintain educational validity in the eyes of the public, the term "outdoor education" was used to emphasize that the programs were focused on the education that took place, not the camping itself (Ford, 1981). The roots of outdoor education as a general concept cannot be traced to any one person or event in history but has been mentioned as early as 3000 B.C. when the Egyptian King Thames stated that true learning must be based on experiences of the real world (Ford, 1986). Originally, humans spent almost their entire lives outside and therefore all of their experiences and education occurred outdoors. Modern outdoor education could be thought of as a means of compensation for a lack of interaction with the outdoor environment as movement is made toward a more developed and urban world.

From the beginning, researchers and practitioners of outdoor education have documented many educational, personal, and interpersonal outcomes associated with participation in outdoor education programs. Unlike classroom learning, outdoor education fosters "connected knowing," where education is part of, rather than separate from, daily life (Gardner, 1991). An analysis of Outward Bound programs found that outdoor education stimulates development of interpersonal skills, leadership skills, and 
has positive effects on sense of empowerment, self-control, independence, selfunderstanding, assertiveness, and decision making skills (Hattie et al., 1997). This metaanalysis examined literature related to outdoor education as a whole and found 40 documented outcomes which were categorized as leadership, self-concept, academic, personality, interpersonal, and adventurousness (Hattie et al., 1997). Outdoor education instills a connectedness with nature and the wider community that helps develop an awareness of relationships with others in the community (Fouhey \& Saltmarsh, 1996).

\section{Outdoor Adventure Recreation Programs}

Outdoor adventure recreation programs exist within the larger context of outdoor education. Similar to many other forms of wilderness experiences, adventure recreation activities are essentially non-utilitarian and provide intense, positive, intrinsically enjoyable experiences to participants (Arnould \& Price, 1993). Outdoor adventure programs can vary in length from an afternoon to an entire semester and usually involve groups of 16 people or less. A common element of outdoor adventure programs is experiencing wilderness environments and dealing with factors beyond human control such as weather, animals, or rock-fall. Past research documents the common components of adventure programs as elements of uncertainty, perceived risk, excitement, interaction with nature, and effort (Bunting, 1990; Ewert, 1989; Priest, 1990; Riola \& O'Keefe, 1999).

Participation in these programs usually requires gaining a certain competence level in a variety of technical skills to help address the challenges that may be faced either individually or as a group. Well coordinated adventure programs appeal to 
participants of all skill levels. A study conducted by Todd, Anderson, Young, and Anderson (2002), examined changes in motivation over time for participants of varying ability levels and found that "beginners struggle to achieve, intermediates are drawn by more excitement and risk, advanced participants focus on self actualization, and experts seek new challenges to stay involved" (p. 7). Group leaders for adventure programs often act as more of a facilitator or coach rather than a guide, helping to instill teamwork and self-sufficiency among participants (Hattie et al., 1997).

Adventure recreation can be defined as "recreational activities that contain structural components of real or perceived danger and usually involve a natural environment setting in which the outcome is uncertain but is influenced by the participant" (Ewert \& Hollenhorst, 1995, p. 21). One key element of adventure programming is the use of both perceived and actual risk (Priest, 1990). Actual risk is the realistic potential for an injury, loss, or other negative consequence. Perceived risk is in the eye of the beholder and involves no actual threat of physical harm or other losses. An example of an adventure program activity that involves both actual and perceived risk is rappelling. When taking the appropriate safety measures, the actual risks associated with the course component of rappelling would be minor, such as scraped knees or possible friction burns to the hands. However, certain participants may also perceive additional risks that do not actually exist such as injury or death from falling.

Including risk in adventure programming helps participants develop a sense of self by exploring their own limits, skills, and abilities (Meier, Morash, \& Welton, 1980). Rohnke (1986), suggested risk is the "spice" that makes achieving goals satisfying. Outdoor adventure experiences are catalyzed by this purposeful inclusion of risk or 
danger which increase participants' concentration and adds consequence to decision making (Ewert \& Hollenhorst, 1995).

One rather all-encompassing definition of outdoor adventure education that steps away from the discussion of risk was offered by Cinnamon and Raiola (1991):

One of the most important themes in outdoor adventure education is that the participants should be provided with the necessary skills, both mental and physical, to enable them to experience success in using and preserving the outdoors. The emphasis is not on winning or losing, but rather on facing the challenges of the activity. Some of the generally accepted goals are personal growth, skill development, excitement and stimulation, challenge, group participation and cooperation, and understanding of one's relationship to the natural environment. (p. 130)

The Cinnamon and Raiola quote above mentions many of the outcomes noted in other research but offers one unique comment about the lack of focus on winning or losing. The use of prescribed challenges without an emphasis on winning or losing is a departure from sport, which is a common form of physical challenge for many people. Removing this element of competition or focus on a given outcome, allows participants to focus on the challenge as a personal journey of exploration and self-discovery (Cinnamon $\&$ Raiola, 1991).

Outdoor adventure programs can foster both physical and mental growth for participants. Physically, participants gain fitness, skill, agility, and stamina. Mentally, participants gain technical skills, problem solving ability, determination, and concentration. Sibthorp (2003) determined that adventure programs develop both hard 
skills and life skills that are transferable beyond the course. Emotional benefits for participants can include sense of purpose, self-awareness, and development of self esteem. Extended experiences in wilderness areas can also support the development of environmental ethics and environmentally responsible behavior. Garvey (1999) stated that outdoor adventure programs have the potential to help morally develop students as well.

\section{Outward Bound}

Outward Bound (OB) was founded in 1941 in Aberdovey, Wales as a training program for merchant seamen to give them skills for survival at sea in preparation for the current war (Miner \& Boldt, 1981). The name Outward Bound came from the nautical term "outward bound," which describes the journey of a ship leaving its homeport into the open sea (McKenzie, 2003). The original OB program was based on six core principles that still form the basis of all OB programming: fitness, skill, initiative, perseverance, respect, and service (Martin, 2001). Hahn's original approach to education was both experience-centered and value-centered (Martin, 2001). The current list of Outward Bound core principles contains only one value from Hahn's original OB program, service. Current values include adventure and challenge, inclusion and diversity, social and environmental responsibility, learning through experience, character development, and compassion and service (Outward Bound USA, n.d.). Definitions of OB core values are listed in Table 2.3.

Table 2.3. Outward Bound Core Values (Outward Bound USA, n.d.)

\begin{tabular}{ll}
\hline $\begin{array}{c}\text { Adventure and } \\
\text { Challenge }\end{array}$ & $\begin{array}{l}\text { Challenge is an action that requires special } \\
\text { effort. Adventure is an exciting and remarkable } \\
\text { experience that involves uncertain outcomes and }\end{array}$ \\
\hline
\end{tabular}




\begin{tabular}{|c|c|}
\hline & acceptable risks. \\
\hline $\begin{array}{l}\text { Inclusion and } \\
\text { Diversity }\end{array}$ & $\begin{array}{l}\text { Welcoming and respecting differences } \\
\text { strengthens the richness of ideas and } \\
\text { perspectives brought to bear on challenge and } \\
\text { learning. The transformational experience of } \\
\text { Outward Bound is more powerful when it takes } \\
\text { place in the company of others as diverse as the } \\
\text { society in which we live. }\end{array}$ \\
\hline $\begin{array}{l}\text { Social and } \\
\text { Environmental } \\
\text { Responsibility }\end{array}$ & $\begin{array}{l}\text { Social and environmental responsibility instills } \\
\text { in students a sense of integrity, which results in } \\
\text { choices and actions that have a positive effect on } \\
\text { society and the environment. }\end{array}$ \\
\hline $\begin{array}{l}\text { Learning } \\
\text { Through } \\
\text { Experience }\end{array}$ & $\begin{array}{l}\text { Learning through experience is a lifelong } \\
\text { process based on action and reflection. } \\
\text { Experiences are intentionally designed, } \\
\text { presented and reflected upon to instill values and } \\
\text { promote skill mastery for people at all stages in } \\
\text { their life-span. }\end{array}$ \\
\hline $\begin{array}{c}\text { Character } \\
\text { Development }\end{array}$ & $\begin{array}{l}\text { To enhance self-awareness, self-esteem, } \\
\text { confidence, discipline and motivation, and } \\
\text { improve physical fitness. }\end{array}$ \\
\hline $\begin{array}{l}\text { Compassion } \\
\text { and Service }\end{array}$ & $\begin{array}{l}\text { Compassion is a heartfelt, caring connection to } \\
\text { each other and the world around us. Service is } \\
\text { an active expression of valuing our common } \\
\text { humanity and the natural world. }\end{array}$ \\
\hline
\end{tabular}

In 1962, Hahn bridged the Atlantic, bringing the OB mission to the United States with the formation of The Colorado Outward Bound School in Leadville, Colorado. This was the beginning of the outdoor education movement in North America, introducing the six core values of Hahn's original OB programs, as well as OB's already-well-established focus on quality and safety, dedication to adventure, and making a difference in people's lives (Hirsch, 1999).

Today, Outward Bound International operates over 40 schools in 25 countries on five continents. Within the United States, what had once grown to several independent OB schools has now merged into OB Wilderness. North Carolina Outward Bound School 
is the only $\mathrm{OB}$ program functioning independently of OB Wilderness within the United States (Outward Bound USA, n.d.).

\section{National Outdoor Leadership Schools (NOLS)}

National Outdoor Leadership Schools (NOLS) was founded in 1965 by legendary outdoor educator Paul Petzoldt. After serving as chief instructor for Colorado Outward Bound School for several years, Petzoldt had become frustrated with the limited pool of skilled instructors and sought to create a program to train future leaders in the outdoor education field (Wood, n.d.). The original NOLS facility was located at the rise of the Sinks Canyon outside of Lander, Wyoming at the site of the present day Sinks Canyon State park office (Wood, n.d.).

NOLS is currently recognized as the international leader in the field of wilderness-based education and outdoor leadership. The mission of NOLS is to be the leading source and teacher of wilderness skills and leadership that serve people and the environment. This mission and all NOLS programs are based on six core principles: wilderness, education, leadership, safety, community, and excellence (NOLS, n.d.). These values are explained in Table 2.4.

Table 2.4. NOLS Core Values (NOLS, n.d.).

\begin{tabular}{ll}
\hline Wilderness & We define wilderness as a place where nature is dominant and \\
& situations and their consequences are real. Living in these \\
& conditions, away from the distractions of modern civilization \\
fosters self-reliance, judgment, respect, and a sense of responsibility \\
for our actions. It can also be a profoundly moving experience that \\
leads to inspiration, joy and commitment to an environmental ethic.
\end{tabular}


With this in mind, our courses are designed to help people develop and practice the skills they need to live, travel and play safely in the outdoors. On our expeditions, people learn by accepting and meeting real challenges. Our instructors are educators, not guides. They are committed to inspiring students to explore and develop their understanding of wilderness ethics, leadership, teamwork, natural history, and technical skills.

Leadership We believe that leadership is a skill that can be learned and
practiced. With students and staff, we encourage the evolution of judgment, personal responsibility, and awareness of group needskey leadership traits-through practical experience and timely feedback. We value integrity, experience, accountability, and humility in our leaders.

\begin{tabular}{ll}
\hline Safety & $\begin{array}{l}\text { We accept risk as an integral part of the learning process and of the } \\
\text { environments through which we travel. The recognition and } \\
\text { management of risk is critical to both the development of } \\
\text { leadership and to the safety and health of our students and staff. } \\
\text { We believe successful risk management stems from good judgment } \\
\text { based on experience, training and knowledge. }\end{array}$ \\
\hline NOLS is an international community composed of talented \\
individuals who care deeply about what they do. We value \\
diversity, integrity and personal responsibility while recognizing \\
that our strength lies in teamwork and commitment to our mission \\
and each other. We appreciate creativity, individuality and passion \\
among our staff and as an institution. We take our jobs seriously \\
and pursue our mission with enthusiasm, and we cherish our sense \\
of humor and our ability to laugh at ourselves.
\end{tabular}

For more than 40 years, NOLS has grown from its original summer in 1965 of 100 students to a total of over 75,000 graduates by 2003 (NOLS, n.d.). Although the first summer of NOLS programming in 1965 was strictly male, NOLS has been co-ed since 1966. NOLS currently operates its programs from 11 schools worldwide, and is the largest backcountry permit holder in the United States (NOLS, n.d.). 


\section{Summary}

Means-end theory has evolved from its original application in consumer marketing to an established tool for the assessment of outdoor education and adventure recreation programs. Prior means-end research in the outdoor field has provided examples of data collection methods and lists of attributes, consequences, and values for consideration in this study. The results of previous studies of OB and NOLS outcomes also provide reference points for the selection of content codes and establish a need for a comparative study between the two organizations. Using this initial population for future longitudinal research will allow researchers to make multiple data collections from the same participants over a five year period in order to better understand the impact of their OB or NOLS experience on their life beyond the course.

Outdoor education can be loosely defined as education in, and about, the outdoors. Outdoor adventure recreation programs differ by the purposeful inclusion of either perceived or actual risk as a learning tool. Many outdoor adventure recreation programs encompass curriculum about the outdoors and can be referred to as adventure education.

As two of the largest providers of such programs, OB and NOLS provide an ideal setting to further the application of means-end theory by providing a comparative assessment of the attributes, consequences, and values obtained by participants. Many studies have examined the individual outcomes of OB programs or NOLS programs but very limited research has been conducted to compare the two organizations. The following chapter discusses the methodology that was used for this comparative study. 


\section{CHAPTER 3}

\section{METHODOLOGY}

This chapter explains the purpose of the study, research questions, and the research methods that were used to collect and analyze the data. The subjects for this study were OB and NOLS participants in the Rocky Mountain region during the summer of 2006. Data were collected from $n=510$ subjects. Collecting the data involved the development and testing of an interview script. The means-end analysis is also explained in this chapter.

\section{Study Locales}

This study took place at a total of two OB program sites and one NOLS program site in the Rocky Mountain Region. OB sites included Leadville, Colorado, and Silverton, Colorado. All NOLS data were collected at NOLS headquarters in Lander, Wyoming. Participants were interviewed during the last two days of their course. Both OB and NOLS base camps shared a similar structure for course end days. Course participants spent the last two days arriving at the base camp, sorting and cleaning gear, and participating in a variety of structured activities to emphasize the learning that took place on course. Researchers conducted interviews with participants at idle times during these days including meal periods. 


\section{Research Participants}

Data for this study were collected from a mixture of male and female OB and NOLS participants, ages 14 and above. This study was limited to participants of outdoor adventure courses with a duration of 14 days or more.

Subjects participated in a wide range of activities during their adventure recreation course. Although many courses shared certain activities such as backpacking, orienteering, and leave no trace instruction, each course offered a unique combination of activities creating an incredibly diverse range of experiences when viewing the entire population of subjects.

\section{Instruments Used in Data Collection}

The study utilized a single interview for every participant. The script was originally developed for a study of OB course outcomes (Goldenberg, 2002), and can be viewed in Appendix A. The first section of the interview identified participant demographics, asking for age, ethnicity, and previous OB or NOLS involvement. Choices for ethnicity were limited to Hispanic, African American, American Indian, Asian or Pacific Islander, Caucasian non-Hispanic, and other. Subjects were asked to indicate the duration of the course and activities they participated in on the course.

The remainder of the interview focused on collection of means-end data. Subjects were asked to identify their most memorable or powerful course components and to rank the components in numerical order. Once the subjects had assembled their list of components they were asked a series of laddering questions for each stated component. For each component, the subjects were first asked "why is (component \#1) important to 
you." The researcher then asks the subject, "why is (answer \#1) important to you," and then "why is (answer \#2) important to you," until the subject could no longer associate any more reasons with the current component being discussed. This process of laddering responses and associating course components was repeated for each of the components that the subject had identified in their original list. Collecting data with this method produced multiple "ladders" for each subject. Each ladder represents a participant's thought progression from the attribute (course component) to its associated consequences and values important to that individual.

\section{Procedures}

Subjects were selected using a convenience sampling method. Semi-structured interviews were conducted with participants of OB and NOLS courses on a voluntary basis. The courses selected for the study were chosen based on enrollment and location of course end day. Researchers focused on OB courses in Silverton and Leadville, Colorado, and NOLS courses in Lander, Wyoming due to the large number of potential subjects at each base camp.

Many of the participants in the study $(61.5 \%)$ were minors. The parents of these participants received a consent form in the pre-course packet sent to them by NOLS or OB. This packet included a cover letter and a form that outlined the purpose and methodology of the research and any risks to participation. The instrument, procedures, consent form, and cover letter were approved by the Human Subjects Department at California Polytechnic State University, San Luis Obispo. Parents signed and returned the 
consent form directly to the researchers prior to their course start date. To view the cover letter, please see Appendix B. To view the consent form, please see Appendix C.

The semi-structured interviews were conducted during the last two days of the participants' OB or NOLS course. Many interviews were conducted during meal times and gear sorting and cleaning periods. Researchers remained on-site at the base camp throughout these course end-dates and approached subjects whenever possible. Most interviews took place in the presence of other group members from the course, but some were conducted in a one-to-one setting. Throughout the interview, all responses were recorded by the researcher in the spaces provided on the instrument.

Participants were greeted by the researcher with an explanation of the research being conducted. Participants age 18 or over were given a consent form for their records, minors were identified from a list of completed parental consent forms before being allowed to participate in the study. Participants were given the opportunity to ask any questions about the research or use of the data prior to beginning the interview. While conducting the interview, researchers hand-recorded participants' responses on the preprinted interview script.

\section{Data Analysis}

The data were separated into three categories: laddering data, demographics data, and contact information. Contact information consisted of a self-reported email address, phone number, and mailing address. Participants were encouraged to give as many email addresses and phone numbers as possible. All contact information was kept for future use. Demographics data were entered along with each corresponding ladder. 
The laddering data were analyzed using a three step method. First, the participants' ladders were coded and entered into a data processing computer program known as LadderMap (Gengler \& Reynolds, 1995) by the researchers. Ladder Map is the primary software package for analyzing means-end data. While entering the Ladders into Ladder Map, the researchers developed content codes to categorize the responses by keywords and recurring phrases. The content codes were tested by another researcher to determine intercoder reliability.

The second step in data analysis was the development of an implication matrix. The implication matrix is a tool that helps identify the number of times concepts are linked in the participants' ladders. The matrix shows a complete list of direct and indirect associations among concepts.

The third and final step in the data analysis was the development of hierarchical value maps (HVMs). HVMs are a visual representation of the themes emerging from the data in the implication matrix. HVMs depict concepts within circles linked by lines. The thickness of the lines represents the frequency of the association between those two concepts. The color of the circle depicts what level of a concept it represents; attributes appear white, consequences appear gray, and values appear black.

The HVM represents only the common themes emerging from the data; it does not represent every link made in every participants' ladders. When creating an HVM there are two important considerations that will determine the content and design; the concepts and links addressed by the HVM, and where the concepts should be located relative to the other items of the HVM (Goldenberg et al., 2000). 


\section{Intercoder Reliability}

The data were originally coded by the researchers while being entered into LadderMap software. A representative sample of $25 \%$ of the data was selected by printing the data set and removing every fourth page. The codes were then removed from the ladders in the sample data before being given to an additional researcher who coded the data completely independently. The additional researcher was a student in the Recreation, Parks, and Tourism Administration program at California Polytechnic State University, San Luis Obispo. This researcher was provided with the un-coded ladder data and a list of the attributes, consequences, and values, along with definitions. The re-coded data were compared to the original coded data and found to be in $87.3 \%$ agreement. The final coded data were reviewed by a third graduate student in the Recreation, Parks, and Tourism Administration program at California Polytechnic State University, San Luis Obispo to review discrepancies between the intercoder and the original researcher.

\section{Summary}

The data for this study were collected using semi-structured personal interviews during the summer of 2006. Participants in the study were students in OB and NOLS courses lasting 13 days or longer in the Rocky Mountain regions of Colorado and Wyoming. The interviews took place during the last two days of the participants' OB or NOLS course, at OB and NOLS base camps. Participation in the study was voluntary and subjects were selected using a convenience sampling method.

The interview questions were designed to "ladder" participants' responses and all data were entered into LadderMap software, a program used to analyze means-end data. 
As the data were entered, content codes were developed and then tested by an additional researcher to measure intercoder reliability. From this data, an implication matrix was created to tabulate the frequency of concepts being associated with one another.

Hierarchical value maps (HVMs) were then created to graphically depict the themes and relationships that surfaced in the implication matrix. The following chapter presents the descriptive findings and means-end data from this study. 


\section{CHAPTER 4}

RESULTS

The results from the analysis of this data are presented in two sections: descriptive findings and means-end data. The means-end data represents the participants' responses in the form of attributes, consequences, and values associated with their experiences and the descriptive statistics, including demographics, help further explain the research context.

\section{Descriptive Findings}

A total of 510 interviews were conducted between the two organizations including $31.7 \%(n=162)$ with OB and $68.3 \%(n=348)$ with NOLS. Of the 510 total participants between both organizations, $33.92 \%(n=173)$ were female, and $66.08 \%$ $(n=337)$ were male (see Table 4.1). OB data came from a total of 15 courses, 4 from Marble, CO, 3 from Silverton, CO, and 8 from Leadville, CO. All interviews at OB base camps were conducted in an outdoor setting prior to students re-entering the frontcountry environment.

OB courses used in this study were based in one of the three base camps mentioned previously; Marble, CO, Leadville, CO, and Silverton, CO. Course lengths varied from 14 days to 22 days. A total of $62.96 \%(n=102)$ were male, and $37.04 \%$ $(n=60)$ were female

All NOLS courses used in this study were based in Lander, Wyoming, and varied in length from 14 days to 6 months. Of the 40 total courses included, 37 were open to the 
public and 3 were privately contracted with the US Naval Academy. Of the 348 participants interviewed, $67.53 \%(n=235)$ were male, $32.47 \%(n=113)$ were female.

Table 4.1. Respondents by Organization

\begin{tabular}{lcccccc}
\hline & \multicolumn{2}{c}{ Male } & \multicolumn{2}{c}{ Female } & \multicolumn{2}{c}{ Total } \\
& $f$ & $\%$ & $f$ & $\%$ & $f$ & $\%$ \\
\hline OB & 102 & 20.0 & 60 & 11.7 & 162 & 31.8 \\
NOLS & 235 & 46.1 & 113 & 22.2 & 348 & 68.2 \\
\hline Total & 337 & 66.1 & 173 & 33.9 & 510 & 100 \\
\hline
\end{tabular}

\section{Ethnicity of Participants}

The participants were asked to self report their race or ethnicity and there was very little racial/ethnic diversity. The overwhelming majority $(88.3 \%, n=450)$ were white or Caucasian, 2.4\% ( $n=12)$ were black or African American, 1.9\% (n=9) identified as other, 3.2\% ( $n=16)$ were Asian or Pacific Islander, 4\% $(n=20)$ were Hispanic or Latino, and $.005 \%(n=3)$ did not respond. For a complete list of respondents by Ethnic/Racial group, see Table 4.2.

Table 4.2. Respondents by Ethnic/Racial Group

\begin{tabular}{lcc}
\hline Ethnic group & $\begin{array}{c}\text { Frequency } \\
(n=510)\end{array}$ & $\begin{array}{c}\text { Percentage } \\
(100 \%)\end{array}$ \\
\hline White or Caucasian & 450 & 88.3 \\
Hispanic or Latino & 20 & 3.9 \\
Asian or Pacific Islander & 16 & 3.1 \\
Black or African American & 12 & 2.4 \\
Other & 9 & 1.8 \\
Did not answer & 3 & 0.5 \\
American Indian/Native American & 0 & 0.0 \\
\hline
\end{tabular}




\section{Occupation}

When asked to self-report their occupation, $88.8 \%(n=453)$ of participants were students, $10.5 \%(n=53)$ were non-students, and $0.08 \%(n=4)$ did not respond.

\section{Previous Attendance}

Participants were asked if they had previously attended a course with the same organization (OB or NOLS) in the past. Most participants $(n=465,91.1 \%)$ had not previously attended a course while a small number $(n=41, .08 \%)$ had previously attended a course, and $(n=4,<.01 \%)$ declined to respond.

\section{Course Length}

Course length was reported by course instructors for each group of participants, and only participants of courses 14 days or longer were considered for the study. Most students $(n=260)$ participated in courses ranging from 14-20 days, while $(n=213)$ had courses of 21-27 days, $(n=32)$ had courses of 28 or more days, and $(n=5)$ did not respond. (See Table 4.3)

Table 4.3. Course length

\begin{tabular}{lcc}
\hline & $\begin{array}{c}\text { Frequency } \\
(n=510)\end{array}$ & $\begin{array}{c}\text { Percentage } \\
(100 \%)\end{array}$ \\
\hline $1(14-20$ days $)$ & 260 & 50.9 \\
$2(21-27$ days $)$ & 213 & 41.9 \\
$3(28$ or more days $)$ & 32 & 6.3 \\
Did not respond & 5 & 0.9 \\
\hline
\end{tabular}


Respondents by Age Group

Respondents were asked to report their birth year and based upon their response, researchers coded their age in one of nine categories. Each age group was given a corresponding group number from 1-9. Group one $(n=102)$ consisted of ages 14 and 15 , group two ( $n=205)$ was 16 and 17, group four $(n=46)$ was 18 and 19 , group five $(n=36)$ was 22-25, group six $(n=16)$ was 26-30, group seven $(n=2)$ was 31-40, group eight $(n=8)$ was 41-49, and group nine $(n=5)$ was participants 50 years old or older. For a complete list of age groups and their corresponding number of participants, see Table 4.4.

Table 4.4. Respondents by Age Group

\begin{tabular}{lccc}
\hline Age group & Age range & $\begin{array}{c}\text { Frequency } \\
(n=510)\end{array}$ & $\begin{array}{c}\text { Percentage } \\
(100 \%)\end{array}$ \\
\hline 1 & $14-15$ & 102 & 20.00 \\
2 & $16-17$ & 205 & 41.50 \\
3 & $18-19$ & 86 & 17.00 \\
4 & $20-21$ & 46 & 9.50 \\
5 & $22-25$ & 36 & 7.00 \\
6 & $26-30$ & 16 & 3.10 \\
7 & $31-40$ & 2 & .03 \\
8 & $41-49$ & 8 & 1.70 \\
9 & $50+$ & 5 & .10 \\
Did not respond & & 4 & .07 \\
\hline
\end{tabular}

Recommending OB/NOLS to a Friend

Participants were asked if they would recommend a course with their respective organization (OB or NOLS) to a friend. An overwhelming majority $(99.02 \%, n=505)$ said yes, while $<0.01 \%(n=1)$ said no, and $4(<0.01 \%)$ did not respond. 


\section{Means-End Data}

The second section of the interview collected the means-end data. A total of 41 content codes were developed to categorize participant responses: 16 attributes, 17 consequences, and 8 values. The most frequently mentioned attributes were expeditioning ( $n=331)$, group $(n=260)$, climbing $(n=167)$, and wilderness $(n=123)$. The most frequently mentioned consequences were interactions $(n=389)$, being challenged ( $n=269)$, new experience/opportunity $(n=259)$, and hard skill development $(n=220)$. Values included transference $(n=383)$, sense of accomplishment $(n=271)$, selfrespect/esteem/confidence $(n=245)$, and warm relationships with others $(n=152)$. For a complete alphabetical listing of content codes, see Appendix D. For content codes by frequency, see Appendix E. The content codes were tested by an additional researcher and intercoder reliability was determined to be $87.3 \%$. The following sections explain how the coded data were used to create implication matrixes and hierarchical value maps to help examine the interrelationships of the attributes, consequences, and values.

\section{Implication Matrix}

The next step in the data analysis was the creation of the implication matrix. The implication matrix is a tool that helps identify the number of times concepts are linked in the participants' ladders. The matrix shows a complete list of direct and indirect associations among concepts. For example, in the matrix below for all participants, a reader can see that the attribute of expeditioning was linked to the consequence of being challenged 103 times. A different matrix is created for each combination of variables to 
be studied. For example, an implication matrix could be created to include all data while another may focus only on NOLS females. The implication matrix for all participants can be found in Table 4.5 below. 
Table 4.5 Implication Matrix for All Participants $(n=510)$

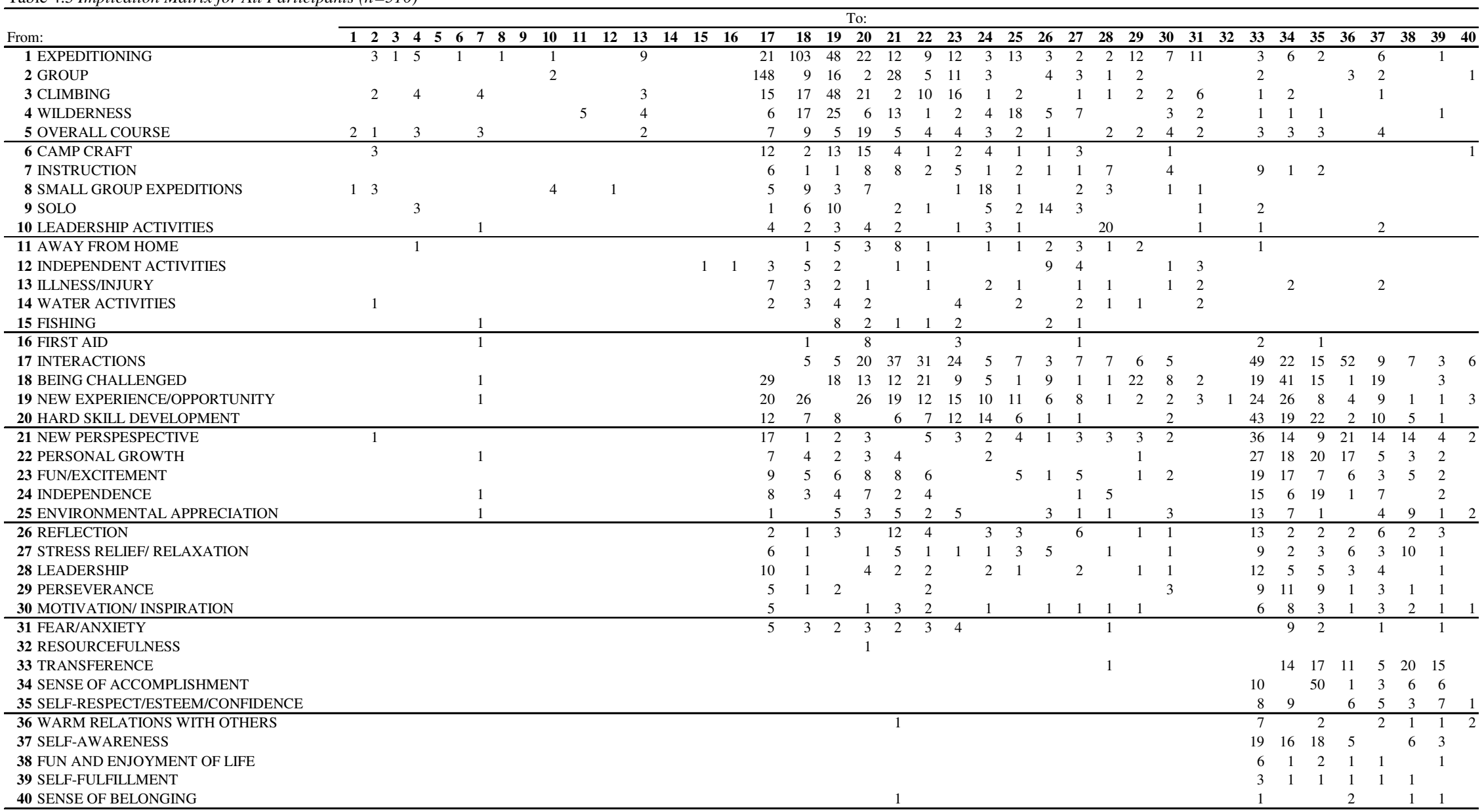




\section{Hierarchical Value Maps}

Once the implication matrix is complete, the next step toward creating a hierarchical value map (HVM) is determining a cut-off value. The cut-off value represents the minimum number of times two concepts need to be linked in order to appear on the HVM. For example a cut-off value of 4 means that the link will not appear on the HVM unless 4 or more participants mentioned the link in their interviews. Cut-off values for this study ranged from 3-8 depending on the size of the subset of data being analyzed. When comparing the cut-off values used in this study and total size of the data subsets they were derived from, the percentages vary from $3.1 \%$ to $6.6 \%$, supporting the $5 \%$ rule of thumb (Gengler \& Reynolds, 1995). A total of seven HVMs were created for this study; one representing all participants, all NOLS, all OB, NOLS female, NOLS male, OB female, and OB male. Cut off value data for each HVM can be found in Table 4.6.

Table 4.6. Cutoff Levels and Percent of Association's for HVMs

\begin{tabular}{llcc}
\hline & Subgroups: & Cut off level & $\begin{array}{c}\text { Percentage of } \\
\text { Associations }\end{array}$ \\
\hline Figure 4.1 & All Participants $(\mathrm{n}=510)$ & 8 & 77.4 \\
Figure 4.2 & NOLS Participants (n=348) & 6 & 80.5 \\
Figure 4.3 & OB Participants (n=162) & 5 & 86.8 \\
Figure 4.4 & NOLS Females (n=113) & 3 & 87.2 \\
Figure 4.5 & OB Females (n=60) & 3 & 78.0 \\
Figure 4.6 & OB Males (n=102) & 4 & 93.6 \\
Figure 4.7 & NOLS Males (n=235) & 4 & 88.0 \\
\hline
\end{tabular}

\section{All participants.}

The HVM for all participants provides a visual representation of the data from the entire study. This HVM had expeditioning $(n=252)$ and group $(n=231)$ as the most frequently mentioned attributes, interactions $(n=297)$, being challenged $(n=211)$, and new experience $(n=199)$ as the most frequently mentioned consequences, and transference $(n=284)$ and sense of 
accomplishment $(n=209)$ as the most frequent values. Nine of the 14 attributes were strongly linked to the consequence of new experience. The consequence of interactions had links to hard skill development, stress relief, sense of belonging, being challenged, and reflection. As with most HVMs from this study, transference was the dominant value and was strongly correlated with several other higher values such as fun and enjoyment of life, warm relationships with others, and self-fulfillment. Fear and anxiety was also linked to sense of accomplishment, which was strongly linked with self respect/esteem/confidence. See Figure 4.1. 


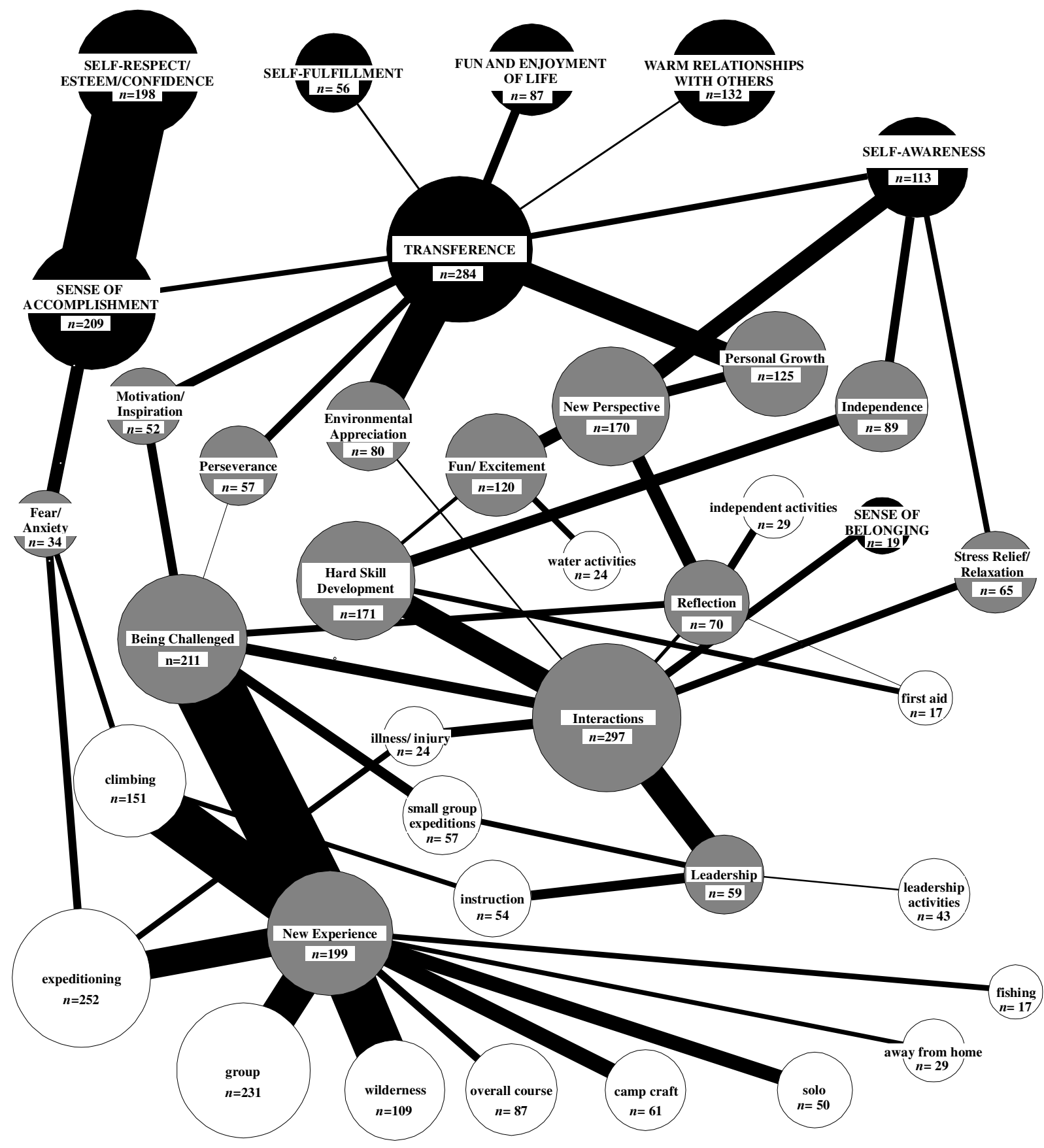

Figure 4.1. Hierarchical Value Map for All Participants $(n=510)$ 


\section{NOLS participants.}

The HVM for all NOLS participants was very similar to that of all participants. The most frequently mentioned attributes were expeditioning $(n=136)$, group $(n=136)$, and climbing $(n=113)$, consequences were interactions $(n=193)$, new experience $(n=125)$, and being challenged $(n=115)$, and values were transference $(n=189)$, self respect/esteem/confidence $(n=131)$, and sense of accomplishment $(n=124)$. As with other HVMs, transference was a key value that also led to other values such as fun and enjoyment of life, warm relationships with others, and self-fulfillment. Both interactions and new experience also served as central hubs, strongly linked to several attributes and consequences. See Figure 4.2. 


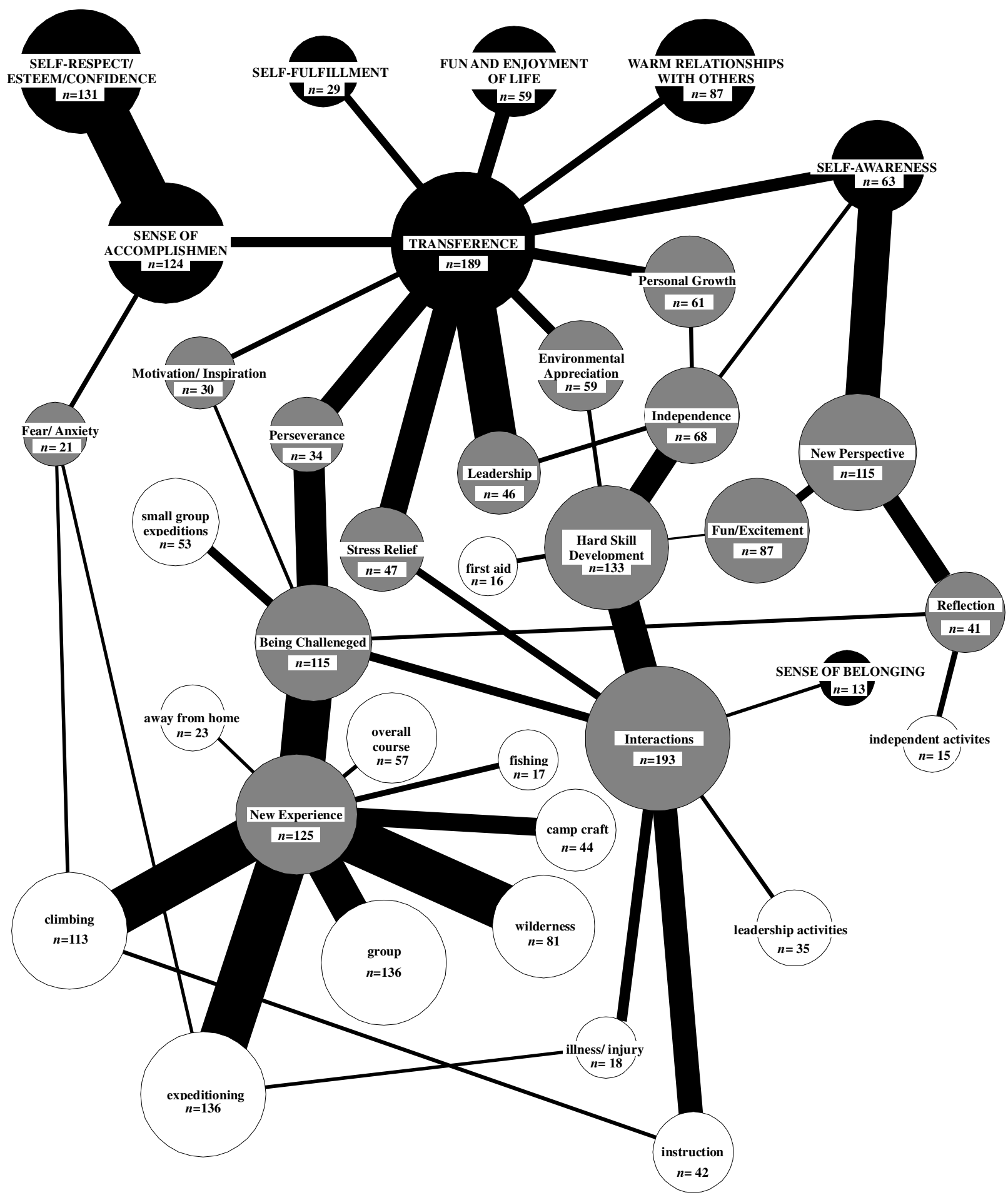

Figure 4.2. Hierarchical Value Map for All NOLS Participants $(n=348)$ 


\section{All OB participants.}

The HVM for all OB participants was very similar to that of all NOLS participants and all participants. This HVM had expeditioning $(n=110)$, group $(n=93)$, and solo $(n=44)$ as the most frequently mentioned attributes. Interactions $(n=102)$, being challenged $(n=91)$, and new experience $(n=70)$ were the most frequently mentioned consequences and transference $(n=93)$, sense of accomplishment $(n=81)$, and self respect/esteem/confidence $(n=64)$ as the most frequently mentioned values. As with many other HVMs from this study, the consequence of new experience and the value of transference were both linked with several other concepts, forming a hub of associations. The key unique feature of this HVM was the strong presence of solo as an attribute. As a uniquely-OB course component, solo, represents an experience on the course that cannot be directly compared to NOLS. See Figure 4.3. 


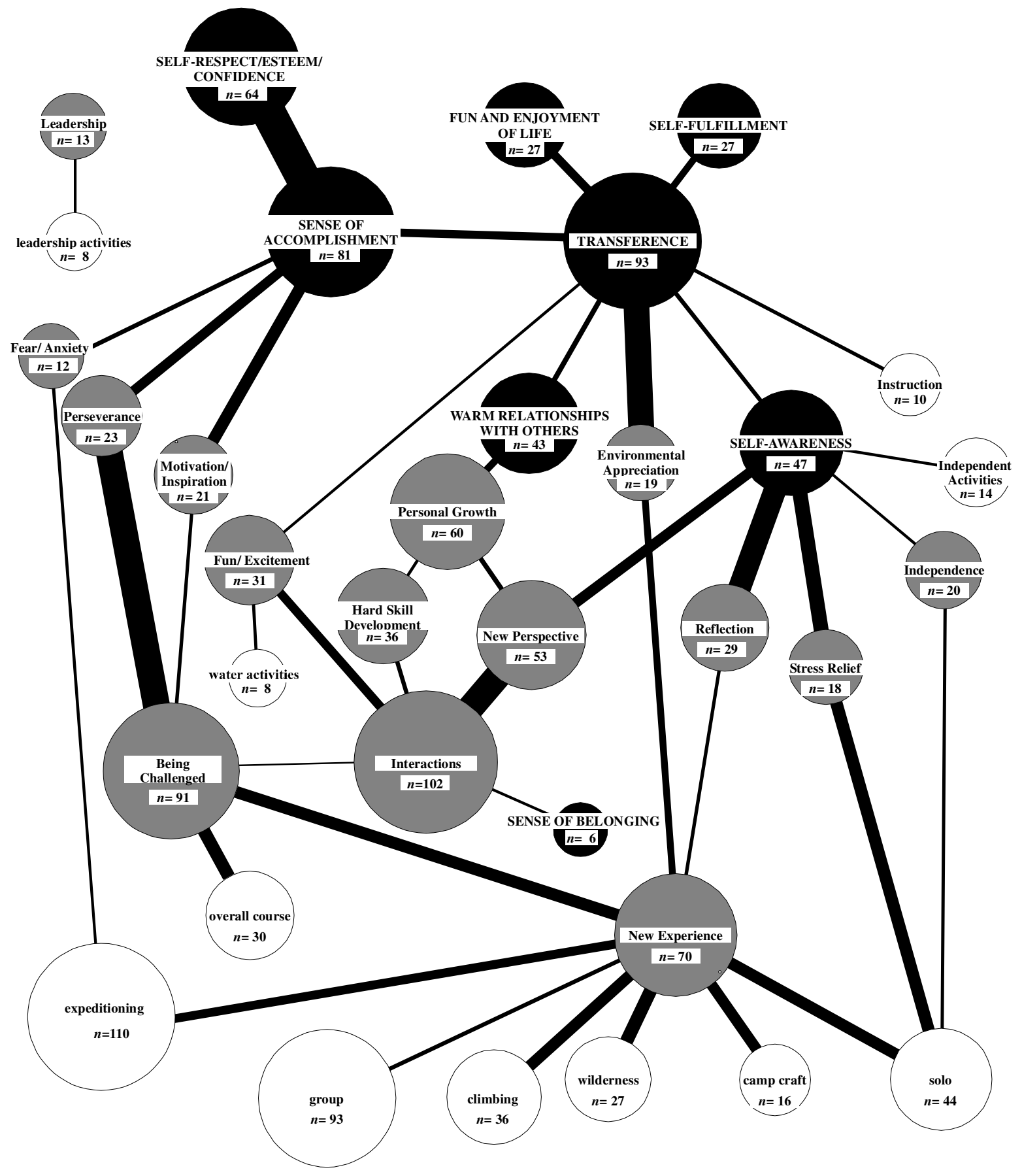

Figure 4.3. Hierarchical Value Map for all OB Participants $(n=162)$ 


\section{NOLS female participants.}

The HVM for NOLS female participants had many similar themes to the HVMs for both organizations yet lines appear much thinner due to the smaller number of participants in this category. Group ( $n=59)$, expeditioning $(n=49)$, and climbing $(n=39)$, were the most frequently mentioned attributes. The most frequently mentioned consequences were interactions $(n=76)$, being challenged $(n=56)$, and new perspective $(n=41)$, values were transference $(n=56)$, self respect/esteem/confidence $(n=50)$, and sense of accomplishment $(n=46)$. Unlike most HVMs in the study which had certain concepts serving as central hubs which were linked to a variety of other concepts, the NOLS female HVM has one very pronounced linear chain of concepts linking expeditioning to new experience, which then lead to being challenged, to interactions, which was linked with hard skill development, which led to fun and excitement, and to new perspective, and ultimately ended with self-awareness. Fear and anxiety was also linked with sense of accomplishment, which was very strongly linked with self respect/esteem/confidence. See Figure 4.4. 


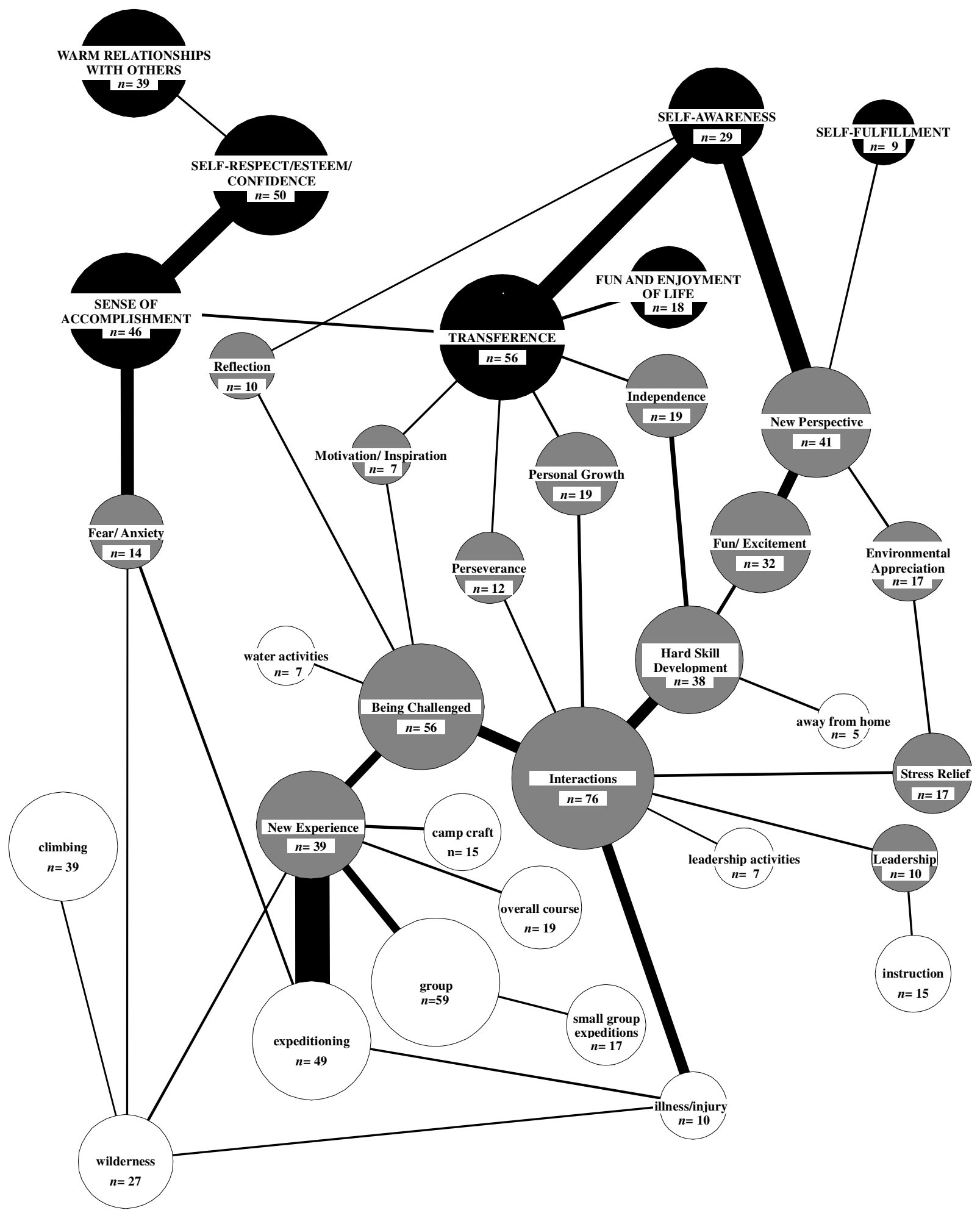

Figure 4.4. Hierarchical Value Map for NOLS Female Participants $(n=113)$ 


\section{OB female participants.}

The HVM for OB female participants had the smallest sample size of any HVM $(n=62)$. Expeditioning $(n=40)$, and group $(n=37)$, were the most frequently mentioned attributes, interactions $(n=42)$, and being challenged $(n=35)$ were the most frequently mentioned consequences, and sense of accomplishment $(n=32)$, transference $(n=29)$, and self respect/esteem/confidence $(n=28)$ were the most frequent values. The consequence of being challenged acted as a central hub and was linked to ten other concepts including 6 attributes and 4 consequences. This HVM did not demonstrate any long linear chains, as were seen in the HVM for NOLS females. See Figure 4.5. 


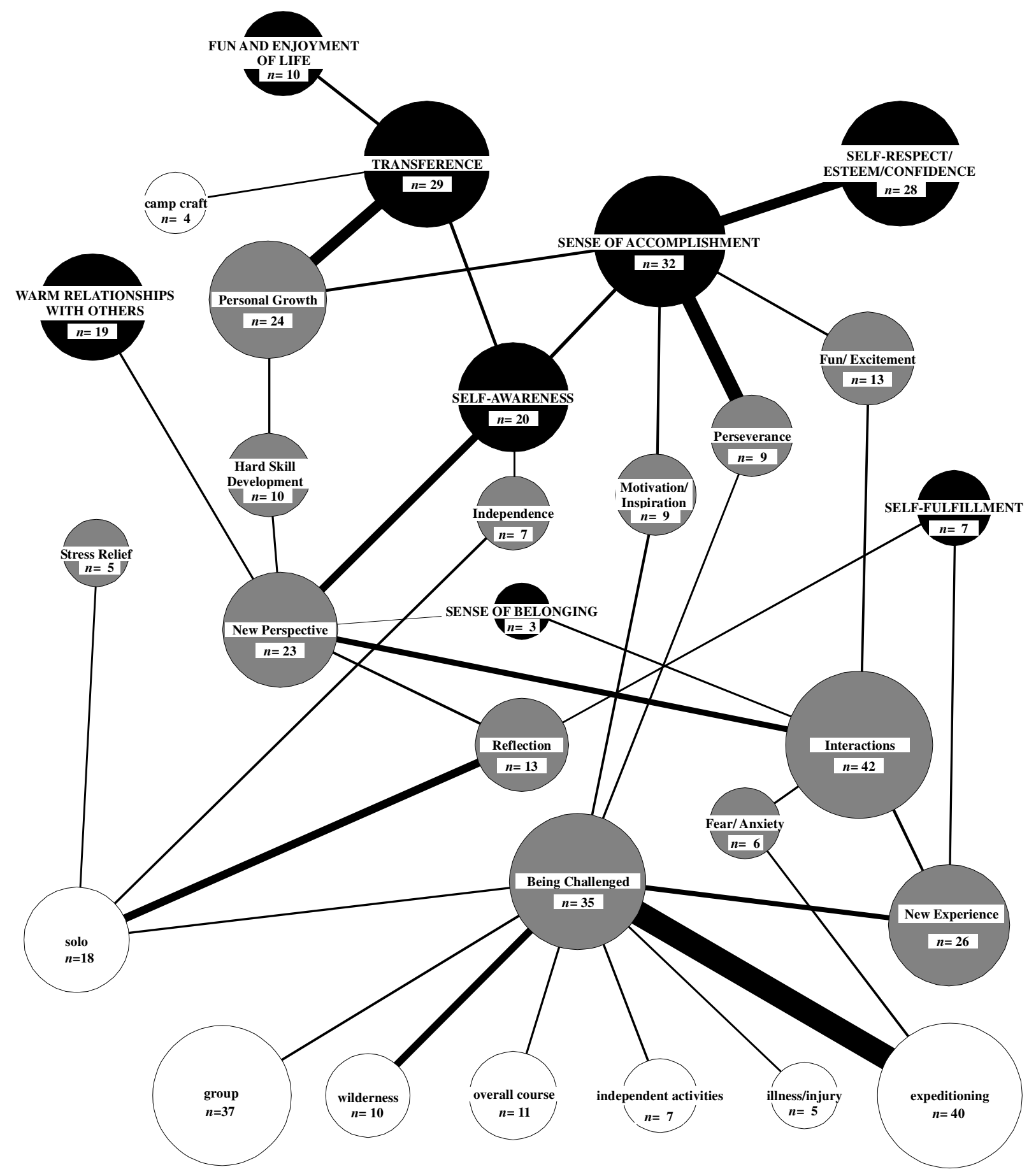

Figure 4.5. Hierarchical Value Map for OB Female Participants $(n=60)$ 


\section{OB male participants.}

The HVM for OB male participants demonstrates both long linear chains of concepts as well as individual concepts serving as a hub for many other concepts. In this case, the value of transference was directly linked to 10 other concepts. As with other HVMs, the consequence of being challenged was linked to interactions which were linked to both new perseverance and personal growth, all of which ultimately led to the value of warm relationships with others. The consequence of being challenged also led to perseverance and the value of self respect/esteem/confidence. The most frequently mentioned attributes were expeditioning $(n=69)$, group $(n=55)$ and climbing $(n=30)$. The most frequently mentioned consequences were interactions $(n=60)$, being challenged $(n=55)$, and new experience $(n=43)$, and the values were transference $(n=63)$, sense of accomplishment $(n=48)$, and self respect/esteem/confidence $(n=36)$. See Figure 4.6. 


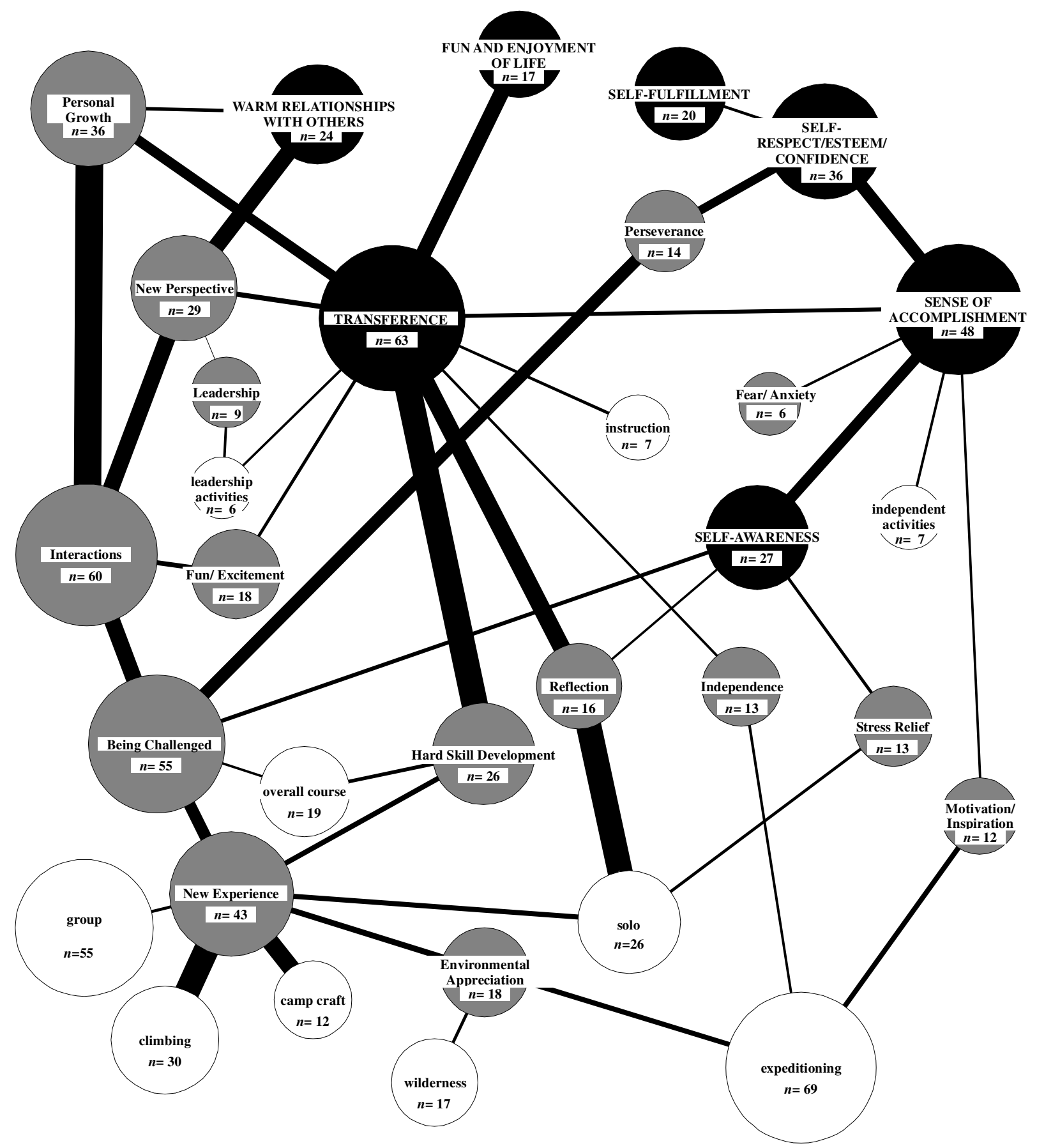

Figure 4.6. Hierarchical Value Map for OB Male Participants $(n=102)$ 


\section{NOLS male participants.}

The HVM for NOLS male participants had many concepts forming central points in the figure which were linked to and from many other concepts, but lacked the long linear chains seen in OB male participants. Expeditioning $(n=87)$, group $(n=77)$, and climbing $(n=74)$ were the most frequently mentioned attributes. Interactions $(n=117)$, hard skills development $(n=95)$, and new experience $(n=86)$ were the most common consequences and transference $(n=133)$, self respect/esteem/confidence ( $n=81)$, and sense of accomplishment $(n=78)$ as the most frequently mentioned values. Transference was not only the most frequently mentioned value, it was also very strongly linked to all other values except for sense of belonging. The consequence of new experience was strongly linked with eight other attributes and consequences, including a very strong link with being challenged. As in other HVMs in the study, the value of sense of accomplishment was very strongly linked to self respect/esteem/confidence, as seen in Figure 4.7. 


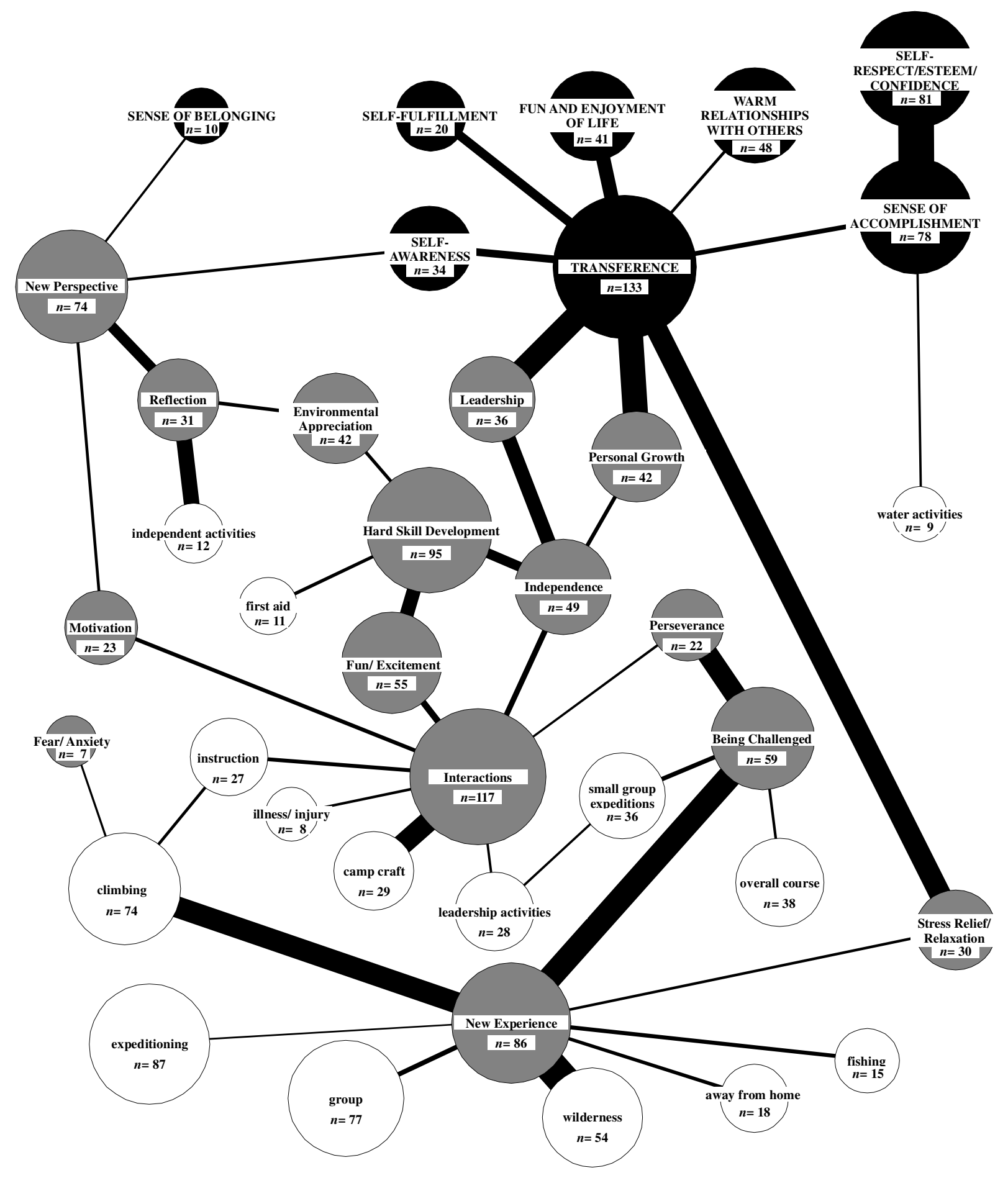

Figure 4.7. Hierarchical Value Map for NOLS Male Participants $(n=235)$ 


\section{Summary}

Data for this study were collected from 510 participants and analyzed using LadderMap software. A system of content coding was utilized and checked by a blind test to determine intercoder reliability. Descriptive statistics show that $68.3 \%$ of participants were from NOLS, $66.08 \%$ were male, $88.3 \%$ were Caucasian, and $78.5 \%$ were between the ages of $14-19$ years old.

Means-end data revealed that the most frequently mentioned attributes were expeditioning, group, climbing, and wilderness. The most frequently mentioned consequences were interactions, being challenged, new experience/opportunity, and hard skill development. Values included transference, sense of accomplishment, self-respect/esteem/confidence, and warm relationships with others. The data were used to create seven HVMs representing the participants responses to the interviews conducted. Discussion, analysis, and implications of these results can be found in the following chapter. 


\section{Chapter 5}

\section{DISCUSSION, IMPLICATIONS, AND RECOMMENDATIONS}

Data collected from 510 participants of NOLS and OB courses is analyzed to determine the outcomes of their outdoor adventure experience. Means-end theory is utilized to produce hierarchical value maps examining the attributes consequences and values. This chapter discusses the findings, implications, and recommendations for future research.

\section{Summary of the Study}

This study is intended to better understand the attributes, consequences, and values of two of the country's premier adventure recreation providers, OB, and NOLS. The study specifically compares the attributes, consequences, and values obtained by participants from the two organizations.

The primary purpose was to compare the outcomes associated with participation in OB and NOLS courses using means-end theory. The secondary purpose was to collect data for future longitudinal analyses. This study uses means-end theory to specifically examine the subjects' links between the components of their OB or NOLS course (the means), and the consequences or values they received from the course (the ends). This study also seeks to compare the subgroups of the population separated by gender, OB participants, and NOLS participants.

Semi-structured interviews were conducted in the summer of 2006 with participants of OB and NOLS courses on a voluntary basis using a convenience sampling method. The courses selected for the study were chosen based on enrollment and location of course end-day. Researchers focused primarily on OB courses in Silverton, Marble, and Leadville, Colorado, and 
NOLS courses in Lander, Wyoming due to the large number of potential subjects at each base camp.

The semi-structured interviews were conducted during the last two days of the participants' OB or NOLS course. While conducting the interview, researchers hand-recorded participants' responses on the pre-printed interview script.

\section{Summary of Data Procedures}

The data were separated into three categories: laddering data, demographics data, and contact information. Demographics data were entered into LadderMap along with each corresponding ladder. All contact information was entered into a spreadsheet and kept for future longitudinal studies. Participants from this initial study will be contacted once annually for the next five years to collect further data about the long-term impact of their course experiences.

The laddering data were analyzed using a three step procedure. First the participants' ladders were coded and entered into a data processing computer program known as LadderMap (Gengler \& Reynolds, 1995) by the researchers. While entering the ladders into LadderMap, the researchers developed content codes to categorize the responses by keywords and recurring phrases. The content codes were tested by another researcher to determine an intercoder reliability rating of $87.3 \%$.

Content codes for attributes and consequences were developed largely by the researcher with particular influence from past works of Goldenberg et al. (2000, 2002, 2005). Values for this study were adapted from Kahle's (1983) study which examined 2,264 Americans and established a list of nine values which were strongly correlated with a person's well being. The Kahle study served as a reference point when reviewing participants' responses and developing 
content codes. The values used in this study include transference, sense of accomplishment, self respect/esteem/confidence, warm relationships with others, self-awareness, fun and enjoyment of life, self-fulfillment, and sense of belonging.

The second step in data analysis was the development of an implication matrix. The implication matrix is a tool that helps identify the number of times concepts are linked in the participants' ladders. The matrix shows a complete list of direct and indirect associations among concepts.

The third and final step in the data analysis was the development of hierarchical value maps (HVMs). HVMs are a visual representation of the themes emerging from the data in the implication matrix. HVMs depict concepts within circles linked by lines. The thickness of the lines represents the frequency of the association between those two concepts. The color of the circle depicts what level of a concept it represents; attributes appear white, consequences appear gray, and values appear black.

Cut off values were used to establish the minimum number of times two concepts were linked in order to appear on the HVM. This enables the researcher to determine the amount of associations that will be depicted on the HVM in order to find the balance between excessive detail which may be challenging to interpret and over simplification which may generalize results beyond the desired level. For this study, cut-off values from 3-8 were used in order to display as much data as possible without overwhelming the reader.

\section{Summary of Significant Findings}

This study has produced findings for the fields of adventure recreation as well as meansend theory. Due to the limitations of the study, primarily the convenience sampling 
methodology, the study cannot be generalized but provides a perspective on the experiences had by 510 participants. Seven HVMs were developed to visually present the data for all participants, all NOLS participants, all OB participants, OB males, OB females, NOLS males, and NOLS females. Each of these participant subgroups offers perspectives on the similarities and differences among the greater population of study participants.

Research Questions Addressed by the Study

This study answers three research questions. Data were collected using personal interviews and means-end theory was used to analyze and interpret the results.

Question 1: "What are the attributes, consequences, and values associated with participation in $O B$ and NOLS adventure recreation courses?"

Respondents' answers were coded into 39 total outcomes consisting of 16 attributes, 15 consequences, and 8 values. Respondents were first asked to list the course attributes or specific experiences that stood out in their mind as being the most significant. This initial collection demonstrates which course attributes left the strongest impression in the minds of the participants at the time of course completion and may provide an interesting reference point for future longitudinal data collection.

The most frequently mentioned attributes were expeditioning $(n=331)$, group $(n=260)$, climbing $(n=167)$, and wilderness $(n=123)$. The most frequently mentioned consequences were interactions ( $n=389)$, being challenged $(n=269)$, new experience/opportunity $(n=259)$, and hard skill development $(n=220)$. Values included transference $(n=383)$, sense of accomplishment $(n=271)$, self-respect/esteem/confidence $(n=245)$, and warm relationships with others $(n=152)$. 
For a complete list of attributes, consequences, and values, see Appendix D, for attributes, consequences, and values by frequency, see Appendix E.

Question 2: "What are the means-end relationships between the attributes, consequences, and values?"

The HVMs used in this study demonstrate the frequency of two concepts being linked through the thickness of the line that connects them. This study utilized seven HVMs and several common themes emerged regarding the outcomes and values. All HVMs showed strong links from the attributes of expeditioning, climbing, group, and wilderness, to the consequence of new experience, which suggests the importance of novelty for the participants. The consequence of new experience was very frequently linked to being challenged, which then led to motivation/inspiration or perseverance, and ultimately the value of transference. This suggests that participants found value in the challenges they encountered and felt that they had gained something from it that would impact their lives beyond the course.

The consequence of interactions, which was often the result of the attribute group, frequently led to hard skill development, new perspective, and personal growth, suggesting that the interpersonal experiences, in and of themselves, had an impact on participants. This chain of thought also included reflection in many HVMs and led to the values of self-awareness and transference.

The most common values obtained for all participants were transference, sense of accomplishment, self respect/esteem/confidence, and self-awareness. Warm relationships with others also had a strong presence though it was mentioned more frequently by female participants than their male counterparts. All HVMs clearly show transference as a key value 
obtained for all participants. In every HVM, transference shows strong links to fun and enjoyment of life. Another value with very consistent patterns among all HVMs is sense of accomplishment, which is sometimes a result of fear/anxiety. Sense of accomplishment also leads to the value of self respect/esteem/confidence in every HVM.

Question 3: "What are the differences in means-end structures between participants of different genders and programs?"

HVMs were created for all participants, all OB participants, all NOLS participants, OB males, OB females, NOLS males, and NOLS females. Though all HVMs are heavily varied, some themes emerge by organization. For example, all NOLS participants have slightly more significant links to hard skills development than their OB counterparts. NOLS and OB females state being challenged and interactions as their most significant consequences yet corresponding males have slightly less emphasis on being challenged and more emphasis on new experience.

This study produced more similarities than differences among the various subsets of the population. For example all HVMs show a clear link from multiple attributes to independence, and ultimately to transference and additional values. The HVMs also show that new experiences, being challenged, and group interactions were significant components for all participants. The most common values obtained also demonstrate great similarity among participant demographics. These values include transference, sense of accomplishment, self respect/esteem/confidence, and self-awareness. 


\section{Discussion}

The following section discusses the data from this study and compares it to the existing body of academic research regarding adventure recreation programs and means-end theory.

\section{Outdoor Education and Outdoor Adventure Recreation Literature}

Findings from this study reinforce several of the previously stated outcomes of outdoor education and adventure recreation programs. OB and NOLS programs offer components of both outdoor education and adventure recreation, however, many of the participants' responses identify with attributes offered in adventure recreation.

Previous studies that focus specifically on the outcomes of OB programs such as Hattie et al. (1997), have found that OB outdoor education programs stimulate development of interpersonal and leadership skills, and have positive effects on sense of empowerment, selfcontrol, independence, self-understanding, assertiveness, and decision making skills. The Hattie et al. study examined literature related to outdoor education and found 40 documented outcomes which were categorized under the following six labels: leadership, self-concept, academic, personality, interpersonal, and adventurousness (Hattie et al.). These findings are consistent with the results of this study. Interactions (with others), skill development, personal growth, transference, sense of accomplishment, and self respect/esteem/confidence are some of the most frequently mentioned consequences and values from the participants. Findings that relate to interpersonal skills, environmental awareness, and warm relationships with others are consistent with Fouhey and Saltmarsh's (1996) findings that outdoor education instills a connectedness with nature, and helps develop an awareness of participants' relationship with others in the community. 
The role of risk as a controlled component of the course is significant in the findings of the study. Adventure recreation has been defined as "recreational activities that contain structural components of real or perceived danger and usually involve a natural environment setting in which the outcome is uncertain but is influenced by the participant" (Ewert \& Hollenhorst, 1995, p. 21). Outdoor adventure experiences are catalyzed by this purposeful inclusion of risk or danger that increases participants' concentration and adds consequence to decision making (Ewert \& Hollenhorst, 1995).

Perceived risk, in the form of climbing and snow travel activities, frequently leads to feelings of fear and anxiety in all participant subgroups of this study, which is strongly linked to sense of accomplishment, and ultimately self respect/esteem/confidence. The role of fear/anxiety as a contributor to sense of accomplishment could be interpreted as reinforcing the importance of using actual or perceived risk in adventure recreation experiences. Similar results are also found for incidents of actual risk including lightening, and illness and injury among participants.

Past research has documented the common components of adventure programs as elements of uncertainty, perceived risk, excitement, interaction with nature, and effort (Bunting, 1990; Ewert, 1989; Priest, 1990; Riola \& O’Keefe, 1999). Participation in these programs usually requires acquiring a certain competence level in a variety of technical skills to support the challenges that may be faced either individually or as a group. These findings are supported in this study through the frequency of skill development being linked to creating new opportunities which often leads to fun and enjoyment of life.

Both mental and physical benefits to the participants of this study are consistent with findings from past research for OB and NOLS. The differences of the two organizations are insignificant compared to the common components of an adventure recreation program that they 
both share. Sibthorp (2003) determined that adventure programs may develop both hard skills and life skills that are transferable beyond the course. Emotional benefits stated by Sibthorp, and demonstrated in this study, include self-awareness, and development of self-esteem. Extended experiences in wilderness areas may also support the development of environmental ethics and environmentally responsible behavior, as demonstrated by the frequent mentioning of environmental awareness as an outcome. Garvey (1999) stated that outdoor adventure programs have the potential to help morally develop students. Although the attributes, consequences, and values have been noted in this study, only the future longitudinal analyses from the same population will determine the true impacts on the participants' lives beyond the course, and perhaps allow evaluation of possible moral development. By interviewing the participants once annually, future longitudinal researchers will be able to compare the participants' perceptions of their course experience from year to year and see which attributes, consequences, and values have a lasting impression. This longitudinal analysis will be important in determining the actual lasting impact on participants' lives verses short-term perspectives immediately following the completion of the course.

\section{Means-End Literature}

This study expands the existing research, which uses means-end theory to examine outcomes of outdoor education programs, by specifically comparing the attributes, consequences, and values obtained from OB and NOLS. This study utilizes much of the framework created by Goldenberg's (2005) outcomes associated with specific components of an Outward Bound experience. This methodology was altered to provide a comparative view of NOLS, and create the foundation for future longitudinal studies. Means-end theory has seen 
similar application such as studies of ropes course participation (Goldenberg et al., 2000; Haras et al., 2006), greenway/trail benefits and personal values (Frauman \& Cunningham, 2001), an evaluation of a service component of Outward Bound (Goldenberg et al., 2006), outcomes of Outward Bound participation (Goldenberg et al., 2005), and wilderness participation for those with and without disabilities (McAvoy et al., 2006).

The large sample size for this study $(n=510)$ presents several software challenges for the researcher when using LadderMap, the most common tool for analyzing means-end data. Although the results prove that LadderMap has the capability to process such large data sets, the researcher notes several limitations and suggests that future means-end researchers consider using other software packages, particularly for sample sizes that result in frequency values with three or more digits. Many components of LadderMap limit the character count for frequency numbers to two characters. This limitation is not in the software's ability to process data, it is in the software's ability to display data. This requires a researcher to undergo a great deal of manual tabulation of data in order to present it accurately. In addition to the simple effort this requires, it also introduces another opportunity for human error.

\section{Implications}

\section{Practical Implications}

This research provides practical implications for adventure education practitioners, and could be used in the training of personnel, and promotion of the organization, by better understanding the process of achieving specific outcomes. The commonalities noted between the two organizations suggest a consistency of attributes, consequences, and values despite varying course components. However, it is important to note that the using the same content codes for the 
two organizations may have played a role in the appearance of similarity. For example, if one were to examine the different course components that were labeled as expeditioning for the two organizations, it is possible that one organization would have had a higher percentage of certain components than the other. For the purposes of this study, any differences at that level are undetectable, this may contribute to the appearance of them being similar. However, if content codes were developed completely organically for each organization, the ability to compare could be lost entirely.

The data suggests that being challenged, as well as other challenge-oriented, perhaps negative consequences such as illness/injury, and fear/anxiety, were very powerful components of means-end chains leading to very positive results such as self-awareness, selfrespect/esteem/confidence, and sense of accomplishment. This further reinforces the idea of using elements of risk to push participants out of their comfort zones as a tool for self-discovery and self-improvement. For the practitioner, this may reinforce the need to adapt the challenge to the individuals within the group so that all participants can be challenged appropriately yet remain within the confines of what is determined to be an acceptable level of safety.

These documented attributes, consequences, and values can be used by both organizations in the promotion of their programs and recruitment of new participants. For example, the frequency of values such as self-respect/esteem/confidence and self-awareness could re-enforce the appeal of the programs in the minds of parents who want their children to overcome insecurity and gain a sense of self-awareness. The results of the study provide a powerful examination of the participants' perceived outcomes at the time of course completion and it is likely that the future longitudinal component of the study will further discover the presence of these outcomes, or lack thereof, in their lives beyond the course. 
In addition to using this information to recruit participants, it may also be useful for grant-writing and other fund development purposes. Providing funding organizations with data that support the positive impacts being made on participants lives will help the organizations further justify the benefit their programs provide. The use of HVMs may also assist by allowing interested parties such as funders to not only see the values obtained but also to understand the thought process that leads from specific course attributes to the ultimate values.

\section{Research Implications}

Data from this study support the findings of previous literature regarding outcomes of outdoor education and adventure recreation programs yet also indicate opportunities to further expand the body of available research. This study was designed to provide a comparative view of the two organizations from a broad, general perspective, but the opportunity exists to further examine specific components in much greater detail.

Data from this initial collection was taken from participants in the final days of their OB or NOLS course, which was observed to be a highly emotional experience for many participants. To better understand the lasting impact on participants' lives, this study was designed with the secondary purpose being the collection of data for future longitudinal analysis. Study participants will be interviewed once annually for the following five years in order to better understand the long-term impact of participation in their OB or NOLS courses. This longitudinal analysis will be very important in understanding the true impact of these courses by comparing the responses year after year. It is anticipated that longitudinal analyses will enable researchers to look past emotionally-charged responses taken at a single point in time and assemble a collective body of data taken over a five year period. 
This study involved 510 participants on various trips throughout the greater Rocky Mountain region, and researchers encountered a wide variety of responses, ultimately settling on 40 content codes. Careful selection of content codes is a critical in controlling the focus of the study. Because the goal of this study was to examine the attributes, consequences, and values of the two organizations from a broad, general perspective, content codes were selected which grouped several of the course components into similar categories. For example, an HVM will show the attribute of expeditioning as very significant, and frequently linked to certain consequences and values. However, it is important to note that a specific comment that had been coded as expeditioning could have been any of the following: peak ascents, hiking, backpacking, off trail hiking, orienteering, river crossing, or snow travel. This study was not designed to examine specific course components but the data does exist within the dataset and future researchers could re-code the participants' responses in order to examine specific components in greater detail. For example, re-coding the same dataset to distinguish the individual course activities could allow future researchers to compare all of the attributes, consequences, and values associated with individual course activities and create data to assist with curriculum development.

\section{Final Thoughts}

This study has further expanded the body of research on outcomes of adventure recreation courses and provided useful insight on the consequences and values obtained by participants. Through comparative examination of two of the nation's largest outdoor program providers, OB and NOLS, this research has documented many positive impacts on participants' lives and collected data which can be used for future longitudinal studies. 
This initial dataset, collected by personal interview at the time of course completion, offers a point-in-time examination of the perceived impacts of the participants' experiences, prior to re-entering their normal daily routines. The setting of the interviews is a limitation of the study for a variety of reasons. In order to reach as many participants as possible, some interviews were conducted at course-end points that were located in a natural setting such as a campground while others took place in a base camp environment. These environments varied greatly and each came with their share of distractions such as other group members, other groups, gear de-issuing, meals, and waiting for showers.

Efforts were made to conduct interviews in a one-on-one setting, however many were conducted within hearing distance of other participants. This factor could have influenced participant responses by either withholding information out of fear of other members overhearing or by overhearing previous interviewees and having thoughts influenced by their responses.

Weather was another limitation that was present during the interviews. Some interviews were conducted on warm sunny days and others were conducted huddled under a tarp during heavy rains. Though no trends were noticed by the researcher, it is possible that an impact was made by this variance in the interview setting.

Future data collection from the same study participants in future longitudinal studies will help determine the extent of the impact of adventure recreation courses on their lives and hopefully produce consistent results over the multiple years of collection, therefore diminishing the impact of the interview setting during this initial collection.

Many participants mentioned attributes that occurred toward the end of the course such as the final group debrief or final peak ascent. These may have truly been the most significant components for these participants, but it is also possible that data were impacted by a recency 
bias. In other words, participants may have been inclined to comment on experiences that occurred more recently, simply because they were fresh in their minds.

The semi-structured interviews loosely followed a script that had participants state their "favorite" course components. After the initial test interviews, researchers felt that this choice of words created possible bias toward positive experiences. In an effort to reduce bias, most of the semi-structured interviews altered this language to state some version of "memorable or most powerful components." The use of the word favorite and variances of the semi-structured format may have influenced the participants' responses.

The attributes, consequences, and values used in this study were developed based on previous research, as discussed in previous chapters, and by grouping participant responses into like categories. Because the same attributes, consequences, and values were used for both organizations, it is possible that the choice of attributes, consequences, and values influenced the appearance of similarity among the two organizations. If the content codes were developed completely organically for each organization, it is possible that results may have varied. However, using different attributes, consequences, and values for the two organizations would eliminate any direct comparison.

Though there are many barriers to generalizing this study, the large sample size makes a contribution to the field of outdoor program research by providing a reference point based on the experiences of 510 participants. Future researchers may find useful information in the methodology of the study, if attempting to conduct qualitative research with a large sample size.

This study has examined outdoor education and adventure recreation programs using a sample size much larger than most prior research. Through a detailed analysis of the attributes, consequences, and values perceived by participants, useful information has been obtained that 
can be used by practitioners and researchers alike. It is this researcher's hope that future researchers will continue to build on the existing body of knowledge in order to further advance the effectiveness of such programs and promote the availability to participants who can benefit most. 
REFERENCES 


\section{REFERENCES}

Arnould, E. J., \& Price, L. L. (1993). River Magic: Extraordinary experience and the extended service encounter. Journal of Consumer Research, 20(6) 24-45.

Bagozzi, R. P., \& Dabholkar, P. A. (2000). Discursive psychology: An alternative conceptual foundation to means-end chain theory. Psychology \& Marketing, 17(7), 535-586.

Bagozzi, R., \& Dabholkar, P. (1994). Consumer recycling goals and their effect on decision making to recycle: A means-end chain analysis. Psychology \& Marketing, 11(4), 313340.

Baker, S., Thompson, K., \& Engelken, J., (2002). Mapping the values driving organic food choice: Germany vs the UK. European Journal of Marketing, 38(8), 995-1012.

Barczak, G., Ellen, P. S, \& Pilling, B. K. (1997). Developing typologies of consumer motives for use of technology based banking services. Journal of Business Research, 38, 131-139.

Botschen, G., \& Hemetsberger, A. (1998). Diagnosing means-end structures to determine the degree of potential marketing program standardization. Journal of Business Research, 42, $151-159$.

Brunso, K., Scholderer, J., \& Grunert, K. (2004) Closing the gap between values and behavior-a means-end theory of lifestyle, Journal of Business Research, 57(2004), 665-670.

Bunting, C. (1990). Interdependency: A key in environmental and adventure education. In J. C. Miles \& S. Priest (Eds.), Adventure education (pp. 453-458). State College, PA: Venture.

Cinnamon, J. \& Raiola, E. (1991). Adventure skill and travel modes. In D. Cockrell (Ed.), The Wilderness Educator: The Wilderness Education Association Curriculum Guide (pp. 129-130). Merrillville, IN: ICS books. 
Collen, H., \& Hoekstra, J. (2001). Values as determinants of preferences for housing attributes, Journal of Housing and the Built Environment, 14(3-4), pp. 285-306.

Costa, A., Dekker, M., \& Jongen W. (2004). An overview of means-end theory: potential application in consumer-oriented food product design. Trends in Food Science and Technology, 15(2004), 403-415.

Devlin, D., Birtwistle, G., \& Macedo, N. (2003). Food retail positioning strategy: a means-end chain analysis. British Food Journal, 105(9), 653-670.

Diggle, P. J., Liang, K. Y., \& Zeger, S. L., (1994). Analysis of longitudinal data. Oxford: Oxford University Press.

Ewert, A. (1989). Outdoor adventure pursuits: Foundations, models, and theories. Columbus, OH: Publishing Horizons.

Ewert, A., \& Hollenhorst, S. (1995). Adventure recreation and its implications for wilderness. International Journal of Wilderness, 3(2), 21-16.

Ford, P. (1986). Outdoor education: Definition and philosophy. Retrieved April5, 2006 from http://www.ericfacility.net/ericdigests/ed267941.html.

Ford, P. (1981). Principles and practices of outdoor/environmental education. New York, NY, John Wiley \& Sons.

Fouhey, H., \& Saltmarsh, J. (1996). Outward Bound and community service learning: An experiment in connected knowing. The Journal of Experiential Education, 19(2), 82-89.

Frauman, E., \& Cunningham, P. H. (2001). Using means-end approach to understand the factors that influence greenway use. Journal of Park and Recreation Administration, 19(3), 93113. 
Futopoulos, C., Krystallis, A., \& Ness, M. (2003). Wine produced by organic grapes in Greece: using means'end chains analysis to reveal organic buyers' purchasing motives in comparison to non-buyers. Food Quality and Preference, 14(2003), 549-566.

Garbarino, E., \& Johnson, M. S. (2001), The different roles of satisfaction, trust, and commitment in customer relationships, Journal of Marketing, 63(2), pp. 70-87.

Gardner, H. (1991). The tensions between education and development. Journal of Moral Education, 20(2), 113-125.

Garvey, D. (1999). Outdoor adventure programming and moral development. In, J. C. Miles and S. Priest (Eds.). Adventure programming. (pp. 133-139). State College, PA: Venture Publishing, Inc.

Gengler, C. E., \& Reynolds, T. J. (1995). LadderMap [Computer Software]. Camden, NJ: Means-End Software.

Goldenberg, M. (2002). Understanding the outcomes of outdoor adventure experiences using means-end analysis. Unpublished doctoral dissertation, University of Minnesota.

Goldenberg, M. A., Klenosky, D. B., O’Leary, J. T., \& Templin, T. J. (2000). A means-end investigation of ropes course experiences. Journal of Leisure Research, 32(2), 208-224.

Goldenberg, M., McAvoy, L., \& Klenosky, D. (2005). Outcomes from the components of an Outward Bound experience. Journal of Experiential Education, 28(2), 123-146

Goldenberg, M. A., Pronsolino, D., \& Klenosky, D. B. (2006). An employee perspective of service in an outdoor education organization: A means-end study. Research in Outdoor Education. , 8, 94-104.

Graeff, T. R. (1997). Comprehending product attributes and benefits: The role of product knowledge and means-end chain inferences. Psychology \& Marketing, 14(2), 163-183. 
Grunert, K., \& Bech-Larsen, T. (2004) Explaining choice option attractiveness by beliefs elicited by the laddering method. Journal of Economic Psychology, 26(2005), 223-241.

Grunert, K. G., \& Grunert, S. C. (1995). Measuring subjective meaning structures by the laddering method: Theoretical considerations and methodological problems. International Journal of Research in Marketing, 12, 209-225.

Gutman, J. (1982). A means-end chain model based on consumer categorization processes. Journal of Marketing, 46, 60-72.

Gutman, J., \& Miaoulis, G. (2003). Communicating a quality position in service delivery: an application in higher eduction. Managing Service Quality, 13(2), 105-111.

Haras, K., Bunting, C., \& Witt, P. (2006). Meaningful involvement opportunities in ropes course programs. Journal of Leisure Research, 28(3), 339-362.

Hattie, J., Marsh, H., Neill, J., \& Richards, G. (1997). Adventure education and Outward Bound: Out-of-class experiences that make a lasting difference. Review of Educational Research, 67(1), 43-87.

Herrmann, A., Huber, F., \& Braustein, C. (2000). Market-driven product and service design: Bridging the gap between customer needs, quality management, and customer satisfaction. International Journal of Production Economics, 66, 77-96.

Hirsch, J. (1999). Developmental adventure programs. In J. C. Miles \& S. Priest (Eds.). Adventure programming. (pp. 13-27). State College, PA: Venture Publishing, Inc.

Hofstede, F., Audenaert, A., Steenkamp, J., \& Wedel, M. (1998). An investigation into the association pattern technique as a qualitative approach to measuring means-end chains. International Journal of Research in Marketing, 15, 37-50. 
Hofstede, F., Steenkamp, J., \& Wedel, M. (1999). International market segmentation based on consumer-product relations. Journal of Marketing Research, XXXVI, 1-17.

Jaeger, S., \& MacFie, H. (2001). The effect of advertising format and means-end information on consumer expectations for apples. Food Quality and Preference, 12(2001), 189-205.

Jansen-Verbeke, M., \& van Rekom, J. (1996). Scanning museum visitors: Urban tourism marketing. Annals of Tourism Research, 23(2), 364-375.

Kahle, L. (Ed.). (1983). Social values and social change: Adaptation to life in America. New York, NY: Praeger.

Klenosky, D. B. (2002). The "pull” of tourism destinations: A means-end investigation. Journal of Travel Research, 40, 385-395.

Klenosky, D., Frauman, E., Norman, W., \& Gengler, C. (1998). Nature-based tourists' use of interpretive services: A means-end investigation. The Journal of Tourism Studies, 9(2), 26-36.

Klenosky, D., Gengler, C., \& Mulvey, M. (1993). Understanding the factors influencing ski destination choice: A means-end analytic approach. Journal of Leisure Research, 25(4), 362-379.

Klenosky, D. B., Templin, T. J., \& Troutman, J. A. (2001). Athletic recruiting: A means-end investigation of student athletes' school choice decisions. Journal of Sport Management, 15(2), 95-106.

Langerak, F., Peelen, E., \& Nijssen, E. (1999). A laddering approach to the use of methods and techniques to reduce the cycle time of new-to-the-firm products. Journal of Product Innovation Management, 16, 173-182. 
Lappin, E. (2000). Outdoor education for behavior disturbed students. Retrieved April 5, 2006 from http://www.ericfacility.net/ericdigests/ed261811.html.

Leppard, P., Russell, C., \& Cox, D. (2003). Improving means-end chain studies by using a ranking method to construct hierarchical value maps. Food Quality and Preference 15(2004) 489-497.

Ligas, M. (2000). People, products, and pursuits: Exploring the relationship between consumer goals and product meanings. Psychology \& Marketing, 17(11), 983-1003.

Martin, A. J. (2001). Towards the next generation of experiential education programmes: A case study of Outward Bound. Unpublished doctoral dissertation, Massey University, Palmerston North, New Zealand.

McAvoy, L., Holman, T., Goldenberg, M., \& Klenosky, D. (2006). Wilderness and persons with disabilities: Transferring the benefits to everyday life. International Journal of Wilderness, 12(2), 23-35.

McIntosh, A., \& Thyne, M. (2005). Understanding tourist behavior using means-end theory. Annals of Tourism Research, 32(1) 259-262.

McKenzie, M. (2003). Beyond the Outward Bound process: Rethinking student learning. Journal of Experiential Education, 26(1), 8-23.

Meier, J., Morash, T., \& Welton, G. (1980). High adventure outdoor pursuits, organization and leadership. Columbus, $\mathrm{OH}$ : Publishing Horizons, Inc.

Meyer, B. B., \& Wenger, M. S. (1998). Athletes and adventure education: An empirical investigation. International Journal of Sport Psychology, 29, 243-266.

Miner, J., \& Boldt, J. (1981). Outward Bound U.S.A., learning through experience in adventure-based education. New York: William Morrow and Company, Inc. 
Mort, G., \& Rose, T. (2004). The effect of product type on value linkages in the means-end chain: Implications for theory and method. Journal of Consumer Behavior, 3(3) p. 221.

Mulvey, M. S., Olson, J. C., Celsi, R. L., \& Walker, B. A. (1994). Exploring the relationships between means-end knowledge and involvement. Advances in Consumer Research, 21, $1-7$.

National Outdoor Leadership Schools, (n.d.). NOLS History. Retrieved April 5, 2006, from http://www.NOLS.edu.

Olson, J. C., \& Reynolds, T. J. (1983) Understanding consumers' cognitive structures:

Implications for advertising strategy. In L. Percy \& A. Woodside (Eds.), Advertising and Consumer Psychology (pp. 77-90). Lexington, MA: Lexington Books.

Orsingher, C., \& Marzocchi, G. (2003). Hierarchical representation of satisfactory consumer service experience. Journal of Service Industry Management, 14(2), 200-216.

Outward Bound USA. (n.d.). Retrieved April 5, 2006, from http://www.outwardbound.org

Pieters, R., Baumgartner, H., \& Allen, D. (1995). A means-end chain approach to consumer goal structures. International Journal of Research in Marketing, 12, 227-244.

Pieters, R., Botschen, G., \& Thelen, E. (1998). Customer desire expectations about service employees: An analysis of hierarchical relations. Psychology \& Marketing, 15(8), 755773.

Pitts, R. E., Wong, J. K., \& Whalen, D. J. (1991). Consumers' evaluative structures in two ethical situations: A means-end approach. Journal of Business Research, 22, 119-130.

Priest, S. (1990). Semantics of adventure education. In J. C. Miles \& S. Priest (Eds.), Adventure education (pp.113-117). State College, PA: Venture. 
Reynolds, T. J., \& Gutman, J. (1988). Laddering theory, method, analysis, and interpretation. Journal of Advertising Research, 28(1), 11-31.

Reynolds, T. J., \& Rochon, J. P. (1991). Means-end based advertising research: Copy testing in not strategy assessment. Journal of Business Research, 22, 131-142.

Riola, E., \& O’Keefe, M. (1999). Philosophy in practice: A history of adventure programming. In J. C. Miles \& S. Priest (Eds.), Adventure programming (pp. 45-54). State College, PA: Venture.

Rohnke, K. (1986). Project Adventure: A widely used generic project. Journal of Physical Education, Recreation, and Dance, 57(5), 68-69.

Roth, M. S. (1994). Enhancing consumer involvement in health care: The dynamics of control, empowerment, and trust. Journal of Public Policy \& Marketing, 13(1), 115-132.

Russell, C., Busson, A., Flight, I., Bryan, J., Van Lawick Van Pabst, J., \& Cox, D. (2004). A comparison of three laddering techniques applied to an example of a complex food choice. Food Quality and Preference, 15(2004), 569-583.

Sibthorp, J. (2003). Learning transferable skills through adventure education: The role of an authentic process. Journal of Adventure Education and Outdoor Learning, 3(2), 145-157.

Skytte, H., \& Bove, K. (2004). The concept of retailer value: A means-end chain analaysis. Agribusiness, 20(3), 323-345.

Spreng, R. A., \& Olshavsky, R. W. (1993). A desires congruency model of consumer satisfaction. Journal of the Academy of Marketing Science, 21(3), 169-177.

The Outdoor Institute. (2004) Definitions of outdoor education, development training and recreation. Retrieved April 5, 2006 from: http://www.ukoi.demon.co.uk/Definitions.htm. 
Todd, S. J., Anderson, L., Young, A. B., \& Anderson, D. (2002, April). Differences in motivations over time by level of development: An examination of pre/post adventure recreation experiences. Paper presented at the meeting of the Northeastern Recreation Research Symposium, Bolton Landing, NY.

Walker, B. A., \& Olson, J. C. (1991). Means-end chains: Connecting products with self. Journal of Business Research, 22, 111-118.

Wansink, B. (2003). Using laddering to understand and leverage a brand's equity. Qualitative Market Research, 6(2), 111-118.

Ware, J. H. (1985). Linear models for the analysis of several measurements in longitudinal studies. American Statistician, 39, 95-101.

Wood, H. (n.d.). NOLS History. NOLS website. Retrieved from http://www.nols.edu/about/ history/nols_history.shtml 


\section{APPENDIXES}




\section{$\underline{\text { APPENDIX A }}$ \\ Insights of an Outward Bound/NOLS Participant \\ Interview Script}

Name:

Participation Number:

1. Male or Female

Introduction: Good morning/afternoon/evening. I'm interviewing you on behalf of Outward Bound/NOLS Wilderness and California Polytechnic State University, San Luis Obispo. I am interested in understanding what you got from participating in your Outward Bound/NOLS course, and what it meant to you personally. Would you be willing to participate in a 10-minute interview? Is this a good time to do the interview?

If you agree to participate and are 18 or older, I will need you to sign a consent form. If you agree to participate and are under 18, I will need to verify that your parents signed the consent form they received in your pre-trip packet.

As you know the purpose of this interview is to find out what you got out of your Outward Bound/NOLS course. There are no right or wrong answers to these questions. I want you to feel comfortable talking with me and answering my questions. Please be assured that all of your responses will remain completely confidential. Also, when answering a question please refer only to your most recent Outward Bound/NOLS course rather than any other previous outdoor experiences you might have had. Any questions for me? OK, let's begin?

\section{SECTION 1 - General Questions}

2. What year were you born? 19

3. Which of the following best describes you? (please "X" one)

$\square$ White or Caucasian $\quad \square$ Black or African American $\quad \square$ Asian or Pacific Islander

$\square$ American Indian/Native American $\quad \square$ Hispanic or Latino

$\square$ Other:

4. What is your current occupation? (please "X" one) $\square$ Student $\square$ Other:

5. Have you attended previous Outward Bound/NOLS Courses? $\square$ Yes $\quad \square$ No

6. How many days was your Outward Bound/NOLS course? days

7. Which of the following did you participate in during your Outward Bound/NOLS course? ("X" all that apply)

$\square$ Backpacking $\quad \square$ Canoeing $\quad \square$ Rock Climbing $\quad \square$ Ropes Course $\square$ Solo

$\square$ Service Project $\square$ Personal Challenge Event

$\square$ Other, please list additional activities:

8. Would you recommend an Outward Bound/NOLS course to a friend? (please "X" one) 
$\square$ Yes $\quad \square$ No $\quad$ a. If no, please explain:

9. I am interested in what you feel were your favorite components out of your Outward Bound/NOLS course. Please tell me some of the components that stand out in your mind. Any others? (TRY TO GET AT LEAST 3-4... BUT ALLOW FOR MORE)

List of Components:

Ranking:

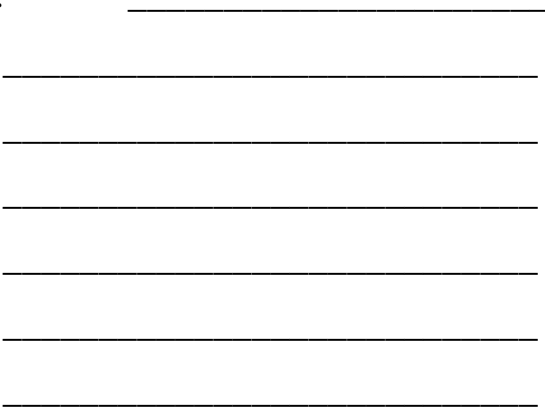

10. Now, I want you to think about the importance of each of these components. Which of the components you mentioned would you say is the most important to you? Which is the next most important? (REPEAT TILL ALL ARE RANKED) 


\section{SECTION 2 - Laddering the Outcomes}

Now, I am going to ask you about some components that you mentioned. You should know that some of my questions will seem obvious or repetitive to you. It is not that I don't understand the obvious, it's just that I need to hear things in your own words to know exactly what you mean. Are you ready to begin?

COMPONENT \#1:

Now you mentioned that (component \#1) was something that you enjoyed about your Outward Bound/NOLS course. Why is important to you? ...And why is that important to you? 
COMPONENT \#2:

Now you mentioned that (component \#2)

your Outward Bound/NOLS course. Why is was something that you enjoyed about important to you? important to you? ... And why is that 
COMPONENT \#3:

Now you mentioned that (component \#3) was something that you enjoyed about your Outward Bound/NOLS course. Why is important to you? ... And why is that important to you? 


\section{APPENDIX B}

\section{Cover Letter}

\section{Dear Outward Bound/NOLS Parents,}

In the coming months, your child will be experiencing the beauty of the Rocky Mountains in an Outward Bound/NOLS Course. They will have the opportunity to experience the weight of a heavy pack, learn the importance of navigation, cooking, communication, and possibly climb granite faces or paddle white-water rapids.

We would love hear your child's feedback upon completion of their course. Your child's perspectives and insights on their Outward Bound/NOLS experience will be very valuable and could be used to evaluate Outward Bound/NOLS programs and to provide successful experiences for participants. Cooperation in this research project from both you and your child is being sought on a purely voluntary basis-you are not required to complete this form. If you choose to allow your child's participation in this study, he or she will be interviewed by a researcher from California Polytechnic State University, San Luis Obispo, on the final day of their course. Interviews will be approximately 10 minutes and will focus on the individual participant's personal outcomes from the course.

Please be assured that your child's identity will remain anonymous and that all responses will be kept completely confidential.

To allow your child's participation in this study, please sign and return the enclosed consent form. On the final day of your child's course, they will be approached by an interviewer and asked to voluntarily participate. The student can choose to not participate at any time. Thank you for your time and assistance. We look forward to learning about your child's Outward Bound/NOLS Experience!

Sincerely,

Marni Goldenberg, PhD.

California Polytechnic State University

Dan Pronsolino

California Polytechnic State University

OB/NOLS Person

OB/NOLS Title

Outward Bound Wilderness/NOLS 


\author{
APPENDIX C \\ Parental Consent Form \\ INFORMED CONSENT TO PARTICIPATE IN \\ Understanding Longitudinal Outcomes of Wilderness \\ Participation Using Means-End Analysis
}

A research project on longitudinal outcomes of wilderness participation using means-end analysis is being conducted by Dr. Marni Goldenberg and Dan Pronsolino, a graduate student, in the Department of Natural Resources Management in the Recreation, Parks, and Tourism Administration Program at Cal Poly, San Luis Obispo. The purpose of the study is to understand the benefits and outcomes associated with participation in the wilderness setting.

Your child is being asked to take part in this study by participating in a 10-minute informal interview. Please be aware that your child is not required to participate in this research and they may discontinue their participation at any time without penalty. They may also omit/not respond to any items in the interview you prefer not to answer.

There are no risks associated with participating in this study.

Your child's responses will be provided confidentially to protect your privacy. Potential benefits associated with the study include a body of research to assist wilderness organizations in understanding and developing awareness towards issues related to long term outcomes associated with wilderness participation.

If you have questions regarding this study or would like to be informed of the results when the study is completed, please feel free to Dr. Marni Goldenberg at (805) 756-7627 or mgoldenb@calpoly.edu. If you have questions or concerns regarding the manner in which the study is conducted, you may contact Steve Davis, Chair of the Cal Poly Human Subjects Committee, at 756-2754, or Susan Opava, Dean of Research and Graduate Programs, at 7561508 .

If you agree to allow your child's voluntary participation in this research project as described, please indicate your agreement by signing below. Please retain this consent form for your reference, and thank you for your participation in this research.

Parent Signature

Date

Parent's Printed Name

Minor's Printed Name 


\section{APPENDIX D}

Alphabetical List of Content Codes and Definitions

$\begin{array}{lll}\text { Attributes } & \text { Consequence } & \text { Values } \\ \text { Away from Home } & \text { Being Challenged } & \text { Fun \& Enjoyment of Life } \\ \text { Camp Craft } & \text { Environmental Appreciation } & \text { Self-Awareness } \\ \text { Climbing } & \text { Fear/Anxiety } & \text { Self-Fulfillment } \\ \text { Expeditioning } & \text { Fun/Excitement } & \text { Self Respect/ Esteem/ Confidence } \\ \text { First Aid } & \text { Independence } & \text { Sense of Accomplishment } \\ \text { Fishing } & \text { Leadership } & \text { Sense of Belonging } \\ \text { Group } & \text { Motivation/Inspiration } & \text { Transference } \\ \text { Illness/Injury } & \text { New Experience/Opportunity } & \text { Warm Relationships with Others } \\ \text { Independent Activities } & \text { New Perspective } & \\ \text { Instruction } & \text { Perseverance } & \\ \text { Leadership Activities } & \text { Personal Growth } & \\ \text { Overall Course } & \text { Reflection } & \\ \text { Small Group Expeditions } & \text { Resourcefulness } & \\ \text { Solo } & \text { Skill Development } & \\ \text { Water Activities } & \text { Stress Relief/Relaxation } \\ \text { Wilderness } & & \end{array}$

Attributes: Characteristics or features of the experience Away from Home

Group

- Missing home

- Away from home for the first time Camp Craft

- Preparing and cooking meals

- Camp chores

- Issues with tent

Climbing

- Rappelling

- Rock climbing

- Multi-pitch rock climbing

Expeditioning

- Hiking

- Backpacking

- Navigation

- River crossing

- Off-trail hiking

- Peak ascent

- Snow travel

First Aid

- WFR Certification and training
- Group experiences/Team activities

- People met/Friends made

- Fellow trip participants

- Volunteer and Environmental service Illness/Injury

- Includes evacuations

Independent Activities

- Activities done alone

- Free time

- 20 mile run

- Differs from OB Solo Experience Instruction

- Course instructors

- Skills learned

- Leave No Trace

Leadership Activities

- Leadership role

- Leader for the Day 
Overall Course

- Overall experience or trip

- Course completion

Water Activities

- Rafting

- Swimming

- Kayaking
Wilderness

- Referring to natural environment

- Weather

- Getting away from urban/suburban environments

Consequences: Benefits and/or perceived risks from course attributes

Being Challenged

- Personal physical/emotional challenges

- Group challenges

- Dealing with frustration

- Differs from perseverance

Environmental Appreciation

- Appreciation or awareness gained for natural environment

Fear/Anxiety

- Being scared or anxious during course

Fun/Excitement

- Feelings of joy or excitement

- Having fun

Independence

- Self-sufficiency

- Doing activities with instructors

- Doing things on one's own

Leadership

- Learning about leadership

- Observing leadership

- Displaying leadership

Motivation/Inspiration

- Feeling motivated or inspired on course

New Experience/Opportunity

- "Physical" experience that was new

New Perspective

- Developing a new perspective
Perseverance

- Preserving through a challenge or difficulty

- Differs from being challenged

Personal Growth

- Growing or maturing because of the course

- Character development

$\circ$ Becoming more outgoing

$\circ$ Being more patient

○ Feeling empowered

Reflection

- Personal reflection of one's growth or maturity during course

Resourcefulness

- Using what was available

- Being creative with available resources

Hard Skill Development

- Using skills learned or developed on course

○ Tying knots

$\circ$ Belaying

- Cooking

Stress Relief/Relaxation

- Getting to relax

- Feelings of relief and relief from stress

- Feelings of comfort 
Values: Participants' desired end-states of

being

Fun and Enjoyment of Life

- Feelings of fun/enjoyment gained from course and applied to life

Self-Awareness

- Awareness of one's own individuality or personality

Self-Fulfillment

- Fulfillment of one's ambitions or desires through one's own efforts Self-Respect/Esteem/Confidence

- Respect: Respect for oneself, character, conduct

- Esteem: Favorable impression of oneself

- Confidence: Confidence in one's own judgment, ability, power, etc.
Sense of Accomplishment

- Feeling good about completing course/task

Sense of Belonging

- Refers to sensing one's place in a group/culture/society/organization

Transference

- Transferring course benefits to another area of one's life

- Future challenges

Warm Relationships w/ Others

- Refers to one's interactions with others

- Being better able to relate to others

- Feeling closer to other

- Altruism (unselfish regard or devotion to other's welfare) 


\section{APPENDIX E}

Content Codes by Frequency

\begin{tabular}{|l|c|}
\hline Attributes & $\boldsymbol{n}=$ \\
\hline Expeditioning & 331 \\
\hline Group & 260 \\
\hline Climbing & 167 \\
\hline Wilderness & 123 \\
\hline Overall Course & 96 \\
\hline Camp Craft & 64 \\
\hline Instruction & 63 \\
\hline $\begin{array}{l}\text { Small Group } \\
\text { Expeditions }\end{array}$ & 60 \\
\hline Solo & 50 \\
\hline $\begin{array}{l}\text { Leadership } \\
\text { Activities }\end{array}$ & 45 \\
\hline Away from Home & 30 \\
\hline $\begin{array}{l}\text { Independent } \\
\text { Activities }\end{array}$ & 30 \\
\hline Illness/Injury & 26 \\
\hline Water Activities & 24 \\
\hline Fishing & 18 \\
\hline First Aid & 17 \\
\hline
\end{tabular}

\begin{tabular}{|l|c|}
\hline Consequence & $\boldsymbol{n}=$ \\
\hline Interactions & 389 \\
\hline Being Challenged & 269 \\
\hline $\begin{array}{l}\text { New } \\
\text { Experience/Opportunity }\end{array}$ & 259 \\
\hline Hard Skill Development & 220 \\
\hline New Perspective & 213 \\
\hline Personal Growth & 144 \\
\hline Fun/Excitement & 138 \\
\hline Independence & 97 \\
\hline $\begin{array}{l}\text { Environmental } \\
\text { Appreciation }\end{array}$ & 87 \\
\hline Reflection & 72 \\
\hline Stress Relief/Relaxation & 70 \\
\hline Leadership & 64 \\
\hline Perseverance & 60 \\
\hline Motivation/Inspiration & 55 \\
\hline Fear/Anxiety & 36 \\
\hline Resourcefulness & 1 \\
\hline
\end{tabular}

\begin{tabular}{|l|c|}
\hline Values & $\boldsymbol{n}=$ \\
\hline Transference & 383 \\
\hline Sense of Accomplishment & 271 \\
\hline $\begin{array}{l}\text { Self- } \\
\text { Respect/Esteem/Confidence }\end{array}$ & 245 \\
\hline $\begin{array}{l}\text { Warm Relationships w/ } \\
\text { Others }\end{array}$ & 152 \\
\hline Self-Awareness & 136 \\
\hline Fun \& Enjoyment of Life & 97 \\
\hline Self-Fulfillment & 65 \\
\hline & 19 \\
\hline Sense of Belonging & \\
\hline
\end{tabular}

